\title{
Riesz transforms of Schrödinger operators on manifolds
}

\author{
Joyce Assaad and El Maati Ouhabaz*
}

\begin{abstract}
We consider Schrödinger operators $A=-\Delta+V$ on $L^{p}(M)$ where $M$ is a complete Riemannian manifold of homogeneous type and $V=$ $V^{+}-V^{-}$is a signed potential. We study boundedness of Riesz transform type operators $\nabla A^{-\frac{1}{2}}$ and $|V|^{\frac{1}{2}} A^{-\frac{1}{2}}$ on $L^{p}(M)$. When $V^{-}$is strongly subcritical with constant $\alpha \in(0,1)$ we prove that such operators are bounded on $L^{p}(M)$ for $p \in\left(p_{0}^{\prime}, 2\right]$ where $p_{0}^{\prime}=1$ if $N \leq 2$, and $p_{0}^{\prime}=\left(\frac{2 N}{(N-2)(1-\sqrt{1-\alpha})}\right)^{\prime} \in(1,2)$ if $N>2$. We also study the case $p>2$. With additional conditions on $V$ and $M$ we obtain boundedness of $\nabla A^{-1 / 2}$ and $|V|^{1 / 2} A^{-1 / 2}$ on $L^{p}(M)$ for $p \in\left(1, \inf \left(q_{1}, N\right)\right)$ where $q_{1}$ is such that $\nabla(-\Delta)^{-\frac{1}{2}}$ is bounded on $L^{r}(M)$ for $r \in\left[2, q_{1}\right)$.
\end{abstract}

keywords: Riesz transforms, Schrödinger operators, off-diagonal estimates, singular operators, Riemannian manifolds.

Mathematics Subject Classification (2010): 42B20, 42B37, 35J10.

\section{Contents}

1 Introduction 2

2 Off-diagonal estimates 5

2.1 Local $L^{2}-L^{q}$ estimates . . . . . . . . . . . . . 6

$2.2 L^{p}-L^{q}$ off-diagonal estimate . . . . . . . . . 8

*Université Bordeaux 1, Institut de Mathématiques (IMB). CNRS UMR 5251.Equipe d'Analyse. 351 Cours de la Libération 33405 Talence, France. joyce.assaad@math.ubordeaux1.fr, Elmaati.Ouhabaz@math.u-bordeaux1.fr 


\section{The Schrödinger operator and the associated Riesz} transforms

3.1 The Schrödinger operator on $L^{2} \ldots \ldots \ldots$. . . . 17

$3.2 \quad L^{p}$ Estimates . . . . . . . . . . . . . . . . 19

$3.3 \quad L^{p}-L^{2}$ off-diagonal estimates of $\nabla e^{-s A}$ and $|V|^{1 / 2} e^{-s A} \quad 20$

3.4 Boundedness of Riesz transforms $\nabla A^{-1 / 2}$ and of $|V|^{1 / 2} A^{-1 / 2}$

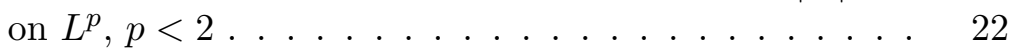

3.5 Boundedness of $V A^{-1}$ and $(-\Delta) A^{-1}$ on $L^{p} \ldots \ldots$

3.6 Boundedness of $\nabla A^{-1 / 2}$ and $|V|^{1 / 2} A^{-1 / 2}$ on $L^{p}$ for $p>227$

\section{Introduction}

Let $M$ be a non-compact complete Riemannian manifold. Denote by $d \mu$ the Riemannian measure, $d$ the geodesic distance on $M$ and $\nabla$ the Riemannian gradient. By $-\Delta$ we denote the positive Laplace-Beltrami operator. We say that $M$ is of homogeneous type if for all $x \in M$ and $r>0$

$$
v(x, 2 r) \leq C v(x, r)
$$

where $v(x, r):=\mu(B(x, r))$ and $B(x, r):=\{y \in M$ such that $d(x, y)<r\}$. This is equivalent to the fact that for some constants $C$ and $N$,

$$
v(x, \lambda r) \leq C \lambda^{N} v(x, r)
$$

for all $\lambda \geq 1$.

Let $p(t, x, y)$ be the heat kernel of the Laplace-Beltrami operator $-\Delta$. We say that $p(t, x, y)$ satisfies a Gaussian upper bound if

$$
p(t, x, y) \leq \frac{C e^{-c \frac{d^{2}(x, y)}{t}}}{v(x, \sqrt{t})}
$$

for all $t>0$, and all $x, y \in M$.

Let us define the Riesz transform $\nabla(-\Delta)^{-1 / 2}$ of the operator $-\Delta$. Since by the Green formula

$$
\|\nabla u\|_{2}=\left\|(-\Delta)^{1 / 2} u\right\|_{2}, \quad \forall u \in W^{1, p}(M),
$$

it follows obviously that $\nabla(-\Delta)^{-1 / 2}$ acts as a bounded operator on $L^{2}$. It is of interest to know if the Riesz transform extends to a bounded operator on $L^{p}$ for $p \neq 2$. When this is the case, we can identify the domain of $(-\Delta)^{1 / 2}$ with the Sobolev space $W^{1, p}$, and obtain $W^{1, p}$-regularity of the solution of the heat equation with initial data in $L^{p}$. We can draw the same conclusions 
if $-\Delta$ is replaced by another self-adjoint differential operator $A$ for which $\nabla A^{-1 / 2}$ acts as a bounded operator on $L^{p}$.

Under the assumptions (1) and $(3)^{1}$ it was proved by Coulhon and Duong [13] that $\nabla(-\Delta)^{-1 / 2}$ is of weak type $(1,1)$ and hence bounded on $L^{p}$ for all $p \in$ $(1,2]$. This quite general result does not hold for other values of $p$; a counterexample is given there showing that the Riesz transform is unbounded on $L^{p}$ for $p>2$. One needs then additional conditions (on the manifold or its heat kernel) to guarantee boundedness of $\nabla(-\Delta)^{-1 / 2}$ on $L^{p}$ for some $p>2$. For this, we refer the reader to Auscher and Coulhon [4], Auscher et al. [5], Carron, Coulhon and Hassell [12], Guillarmou and Hassell [19], [20] and the references therein. Let us also mention the following works of Auscher [2], Blunck and Kunstmann [9], Sikora [27] and Ouhabaz [25] (Chapter 7) dealing with Riesz transforms of elliptic differential operators.

Let us now move to Schrödinger operators on the Euclidean case $M=\mathbb{R}^{N}$. The classical Riesz transform $\nabla(-\Delta)^{-1 / 2}$ is of course bounded on $L^{p}$ for all $p \in(1, \infty)$. If $V$ is a non-negative potential, then $\nabla(-\Delta+V)^{-1 / 2}$ is bounded on $L^{p}$ for all $p \in(1,2]$ (see Ouhabaz [25], Chapter 7 or more general case in Duong, Ouhabaz and Yan [17]) but it may be unbounded on $L^{p}$ for some $p>2$, see Shen [28] for a counter-example. Shen [28], and Auscher and Ben Ali [3] proved that if the non-negative potential $V$ is in the reverse Hölder class $R H_{q}$, then the Riesz transform $\nabla(-\Delta+V)^{-1 / 2}$ is bounded on $L^{p}$ for all $p \in\left(2, p_{1}\right)$ where $p_{1}>2$ depends on $q$. For negative potentials $V$ strongly subcritical with constant $\alpha \in(0,1)$ (i.e. $V$ small with respect to $-\Delta$ in the sense of quadratic forms) the situation is more complicated. Indeed, it is known that the semigroup $\left(e^{-t(-\Delta+V)}\right)_{t>0}$ extends from $L^{2}$ to $L^{p}$ for $p$ in some interval $\left(p_{0}^{\prime}, p_{0}\right)$ and $\left(p_{0}^{\prime}, p_{0}\right)$ is optimal (see Liskevich, Sobol and Vogt [24]). In this setting, Assaad [1] proved that Riesz transform is bounded on $L^{p}\left(\mathbb{R}^{N}\right)$ for $p \in\left(p_{0}^{\prime}, 2\right]$ where $p_{0}^{\prime}$ is the dual exponent of $p_{0}:=\frac{2 N}{(N-2)(1-\sqrt{1-\alpha})}$. If, in addition, $V \in L^{N / 2-\varepsilon}\left(\mathbb{R}^{N}\right) \cap L^{N / 2+\varepsilon}\left(\mathbb{R}^{N}\right), N>2$, for some $\varepsilon>0$, then it is bounded on $L^{p}\left(\mathbb{R}^{N}\right)$ for all $p \in(1, N)$.

For Schrödinger operators $-\Delta+V$ on Riemannian manifolds less is known. If $V$ is non-negative and (1) and (3) are satisfied, then the heat kernel of $-\Delta+V$ is dominated by the heat kernel of $-\Delta$ and hence it satisfies (3). The method of Coulhon and Duong [13], based on a Gaussian upper bound and weighted gradient estimates, works also for $-\Delta+V$ and leads to boundedness of $\nabla(-\Delta+V)^{-1 / 2}$ on $L^{p}$ for $p \in(1,2]$. This also follows from results in Blunck and Kunstmann [9], and Sikora [27]. For $p>2$, Badr and Ben Ali [6] extend the result of Auscher and Ben Ali [3] to the setting of Riemannian

\footnotetext{
${ }^{1}$ when the doubling condition (1) is satisfied, (3) is equivalent to the apparently weaker diagonal estimate: $p(t, x, x) \leq \frac{C}{v(x, \sqrt{t})}$, see Grigor'yan [18], Theorem 1.1.
} 
manifolds which satisfy polynomial volume growth and Poincaré inequalities. As in the Euclidean case, they assume that the potential $V$ is non-negative and belongs to a reverse Hölder class. Note also that the results of Assaad [1] for negative potentials hold in the setting of Riemanniann manifolds which satisfy a global Sobolev inequality. Guillarmou and Hassell [19], [20] studied Riesz transforms $\nabla\left(A \circ P_{+}\right)^{-1 / 2}$ where $A$ is a Schrödinger operator with signed potential and $P_{+}$is the spectral projection on the positive spectrum. They prove in particular that on asymptotically conic manifolds $M$ of dimension $N \geq 3$, if $V$ is smooth and satisfies decay conditions, and the Schrödinger operator has neither zero-modes nor zero-resonances, then the Riesz transform type operator $\nabla\left(A \circ P_{+}\right)^{-1 / 2}$ is bounded on $L^{p}$ for all $p \in(1, N)$.

It is our aim to study the boundedness on $L^{p}(M)$ of Riesz transforms $\nabla(-\Delta+V)^{-1 / 2}$. We consider signed potentials $V=V^{+}-V^{-}$and Riemannian manifolds $M$ for which we merely assume (2) and (3). We do not require any global Sobolev inequality nor smoothness conditions on $V$. We assume that $V^{+} \in L_{l o c}^{1}$ and $V^{-}$is small with respect to $-\Delta+V^{+}$in the sense that

$$
\int_{M} V^{-} u^{2} d \mu \leq \alpha\left[\int_{M}|\nabla u|^{2} d \mu+\int_{M} V^{+} u^{2} d \mu\right],
$$

for all $u \in W^{1,2}(M)$ such that $\int_{M} V^{+} u^{2} d \mu<\infty$, where $\alpha \in[0,1)$. We prove that $\nabla(-\Delta+V)^{-1 / 2}$ and $|V|^{1 / 2}(-\Delta+V)^{-1 / 2}$ are bounded on $L^{p}(M)$ for all $p \in\left(p_{0}^{\prime}, 2\right]$ where $p_{0}^{\prime}=1$ if $N \leq 2$, and $p_{0}^{\prime}=\left(\frac{2 N}{(N-2)(1-\sqrt{1-\alpha})}\right)^{\prime} \in(1,2)$ if $N>2$. The proof is based on techniques from Auscher [2] and Blunck and Kunstmann [8] and [9] together with $L^{p}-L^{q}$ off-diagonal estimates for both of the semigroup $e^{-t(-\Delta+V)}$ and its gradient. We prove in particular estimates of the type

$$
\left\|\chi_{B(x, r)} e^{-s(-\Delta+V)} \chi_{B(y, r)}\right\|_{p-q} \leq \frac{C}{v(x, r)^{\gamma_{p, q}}}\left(\max \left(\frac{r}{\sqrt{s}}, \frac{\sqrt{s}}{r}\right)\right)^{\beta} e^{-c \frac{d^{2}(B(x, r), B(y, r))}{s}},
$$

as well as similar estimates for $\sqrt{s} \nabla e^{-s(-\Delta+V)}$. In order to prove the last estimate we establish a bridge between $L^{p}-L^{q}$ estimates of a semigroup and localized Gagliardo-Nirenberg type inequalities. This is proved in a general setting of metric spaces in the next section.

We also study $\nabla(-\Delta+V)^{-1 / 2}$ and $|V|^{1 / 2}(-\Delta+V)^{-1 / 2}$ with signed potentials on $L^{p}$ for $p>2$. Suppose that for some $r_{1}, r_{2}>2$

$$
\int_{0}^{1}\left\|\frac{V}{v(., \sqrt{s})^{\frac{1}{r_{1}}}}\right\|_{r_{1}} d s+\int_{1}^{\infty}\left\|\frac{V}{v(., \sqrt{s})^{\frac{1}{r_{2}}}}\right\|_{r_{2}} d s<\infty .
$$

We obtain by arguments of perturbation type that $V(-\Delta+V)^{-1}$ and $(-\Delta)(-\Delta+$ $V)^{-1}$ are bounded on $L^{p}$ for all $p \in\left(p_{0}^{\prime}, \frac{p_{0} r}{r+p_{0}}\right)$ if $N>2$ and $p \in(1, r)$ if $N \leq 2$ 
where $r=\inf \left(r_{1}, r_{2}\right)$. If

$$
\int_{0}^{1}\left\|\frac{|V|^{1 / 2}}{v(., \sqrt{s})^{\frac{1}{r_{1}}}}\right\|_{r_{1}} \frac{d s}{\sqrt{s}}+\int_{1}^{\infty}\left\|\frac{|V|^{1 / 2}}{v(., \sqrt{s})^{\frac{1}{r_{2}}}}\right\|_{r_{2}} \frac{d s}{\sqrt{s}}<\infty,
$$

then $(-\Delta)^{1 / 2}(-\Delta+V)^{-1 / 2}$ and $|V|^{1 / 2}(-\Delta+V)^{-1 / 2}$ are bounded on $L^{p}$ for all $p \in(1, r)$ if $N \leq 2$ and $p \in\left(p_{0}^{\prime}, \frac{p_{0} r}{p_{0}+r}\right)$ if $N>2$. In the particular case where $v(x, t) \approx t^{N}$ for some $N>2$, the last condition on $V$ reads as $V \in L^{N / 2-\varepsilon} \cap L^{N / 2+\varepsilon}$ for some $\varepsilon>0$, and the conclusion is that $(-\Delta)^{1 / 2}(-\Delta+V)^{-1 / 2}$ and $|V|^{1 / 2}(-\Delta+V)^{-1 / 2}$ are bounded on $L^{p}$ for $p \in\left(p_{0}^{\prime}, \frac{p_{0} N}{p_{0}+N}\right)$. If in addition Poincaré inequalities hold on $M$, the last interval of $p$ is $(1, N)$.

Notation. In the following sections, we denote by $L^{p}$ the Lebesgue space $L^{p}(M, \mu)$ or $L^{p}\left(M, T^{*} M\right)$ (according to the context) with the Riemannian volume $\mu,\|.\|_{p}$ its usual norm, (.,.) the inner product of $L^{2}$, and $\|.\|_{p-q}$ denotes the norm of operators from $L^{p}$ to $L^{q}$. We set $\gamma_{p, q}:=\frac{1}{p}-\frac{1}{q}$ for $p \leq q$. We denote by $p^{\prime}$ the dual exponent of $p, p^{\prime}:=\frac{p}{p-1}$ and as usual $p^{\prime}=\infty$ if $p=1$. We denote by $C, c$ all inessential positive constants even if their values change at each occurrence. The boundedness of the Riesz transform $\nabla A^{-1 / 2}$ on $L^{p}$ means boundedness from $L^{p}$ of functions into $L^{p}$ of vector fields. Recall also that $v(x, r):=\mu(B(x, r))$ denotes the volume of the ball $B(x, r)$. We shall use the notation $A(x, r, k):=B(x,(k+1) r) \backslash B(x, k r)$, $C_{1}(x, r):=B(x, 4 r), C_{j}(x, r):=B\left(x, 2^{j+1} r\right) \backslash B\left(x, 2^{j} r\right)$ for $j \geq 2$. Finally, $\chi_{E}$ denotes the characteristic function of $E$ and $\chi_{E} S \chi_{F}$ is the operator $f \rightarrow$ $\chi_{E} S\left(\chi_{F} f\right)$.

\section{Off-diagonal estimates}

In order to establish boundedness on $L^{p}$ of the Riesz transform of $-\Delta+V$ we shall need the boundedness of the semigroup $e^{-t(-\Delta+V)}$ on $L^{p}$ together with $L^{p}-L^{q}$ off-diagonal estimates. Upper bounds for the heat kernel of $-\Delta$ (hence $L^{p}-L^{q}$ off-diagonal estimates) is well studied and quite understood subject but such bounds can be destroyed by the presence of a non-trivial negative part of $V$. We shall prove that $L^{p}-L^{q}$ off-diagonal estimates are conserved for $p$ and $q$ in a certain interval around 2. Our approach is based on perturbation arguments. However, since we do not assume $V$ to be bounded, the domains of $-\Delta$ and $-\Delta+V$ are not necessarily the same and therefore properties of $e^{t \Delta}$ do not hold for $e^{-t(-\Delta+V)}$ in a trivial way. The operators here are constructed by the quadratic form technique, we have some information 
on the domains of the square roots $\sqrt{-\Delta}$ and $\sqrt{-\Delta+V}$ and our perturbation technique relies very much on this fact.

The results in the next sub-sections do not use the Riemannian manifold framework and hold for general self-adjoint operators on metric spaces. We shall assume that $A$ is a non-negative self-adjoint operator on $L^{2}(X, \mu, \rho)$ where $(X, \mu, \rho)$ is a metric measured space. Again, $B(x, r)=\{y \in X, \rho(x, y)<$ $r\}$ will denote the open ball with volume $v(x, r):=\mu(B(x, r))$. We may need to assume that $(X, \mu, \rho)$ is of homogenous type which will mean that (2) holds for some constants $C$ and $N$ independent of $x$ and $r$. For simplicity, we write $L^{p}$ instead of $L^{p}(X, \mu)$.

\subsection{Local $L^{2}-L^{q}$ estimates}

The following simple proposition will play an important role in our analysis of off-diagonal estimates. It connects local $L^{2}-L^{q}$ estimates to GagliardoNirenberg type inequalities.

Proposition 2.1. Let $A$ be a non-negative self-adjoint operator on $L^{2}$. Consider the following properties in which $N$ is a positive constant.

i) $\left\|\chi_{B(x, \sqrt{t})} e^{-s A}\right\|_{2-q} \leq \frac{C}{v(x, \sqrt{t})^{\gamma_{2, q}}}\left(\frac{t}{s}\right)^{\frac{N}{2} \gamma_{2, q}}$ for all $0<s \leq t$, and all $x \in X$.

ii) $\left\|\chi_{B(x, r)} u\right\|_{q} \leq \frac{C}{v(x, r)^{\gamma 2, q}}\left[\|u\|_{2}+r\left\|A^{1 / 2} u\right\|_{2}\right]$ for all $r>0$, all $x \in X$, and all $u \in D\left(A^{1 / 2}\right)$.

iii) $\left\|\chi_{B(x, \sqrt{t})} e^{-s A}\right\|_{2-q} \leq \frac{C}{v(x, \sqrt{t})^{\gamma_{2, q}}} \max \left(1,\left(\frac{t}{s}\right)^{1 / 2}\right)$ for all $0<s, t$, and all $x \in X$.

Here $q \in\left[2, \frac{2 N}{N-2}\right)$ if $N>2, q \in[2, \infty)$ if $N=2$ and $q \in[2, \infty]$ if $N<2$. Then $i) \Rightarrow i i) \Rightarrow i i i)$.

Proof: Let us first prove that $i$ ) implies $i i)$. For every $u \in D\left(A^{1 / 2}\right)$, we write

$$
u=e^{-t A} u+\int_{0}^{t} A e^{-s A} u d s=e^{-t A} u+\int_{0}^{t} e^{-\frac{s A}{2}} A^{1 / 2} e^{-\frac{s A}{2}} A^{1 / 2} u d s .
$$

Then

$$
\chi_{B(x, \sqrt{t})} u=\chi_{B(x, \sqrt{t})} e^{-t A} u+\int_{0}^{t} \chi_{B(x, \sqrt{t})} e^{-\frac{s A}{2}} A^{1 / 2} e^{-\frac{s A}{2}} A^{1 / 2} u d s .
$$


Now using $i$ ) and the fact that the semigroup is bounded analytic on $L^{2}$, we have

$$
\begin{aligned}
\left\|\chi_{B(x, \sqrt{t})} u\right\|_{q} & \leq\left\|\chi_{B(x, \sqrt{t})} e^{-t A} u\right\|_{q}+\int_{0}^{t}\left\|\chi_{B(x, \sqrt{t})} e^{-\frac{s A}{2}} A^{1 / 2} e^{-\frac{s A}{2}} A^{1 / 2} u\right\|_{q} d s \\
& \leq \frac{C\|u\|_{2}}{v(x, \sqrt{t})^{\gamma_{2, q}}}+\frac{C}{v(x, \sqrt{t})^{\gamma_{2, q}}} \int_{0}^{t}\left(\frac{t}{s}\right)^{\frac{N}{2} \gamma_{2, q}}\left\|A^{1 / 2} e^{-\frac{s A}{2}} A^{1 / 2} u\right\|_{2} d s \\
& \leq \frac{C}{v(x, \sqrt{t})^{\gamma_{2, q}}}\left[\|u\|_{2}+t^{\frac{N}{2} \gamma_{2, q}}\left\|A^{1 / 2} u\right\|_{2} \int_{0}^{t} s^{-\frac{1}{2}-\frac{N}{2} \gamma_{2, q}} d s\right] .
\end{aligned}
$$

The last integral converges if $\gamma_{2, q}:=\frac{1}{2}-\frac{1}{q}<\frac{1}{N}$. This gives the conditions on $q$ as in the proposition. Therefore we deduce that $i i$ ) holds where $r=\sqrt{t}$. Assume now that $i i$ ) holds. Setting $r=\sqrt{t}, u=e^{-s A} f$ for $f \in L^{2}$ and $s, t>0$ we obtain

$$
\begin{aligned}
\left\|\chi_{B(x, \sqrt{t})} e^{-s A} f\right\|_{q} & \leq \frac{C}{v(x, \sqrt{t})^{\gamma_{2, q}}}\left[\|f\|_{2}+\sqrt{t}\left\|A^{1 / 2} e^{-s A} f\right\|_{2}\right] \\
& \leq \frac{C}{v(x, \sqrt{t})^{\gamma_{2, q}}}\left[1+\left(\frac{t}{s}\right)^{1 / 2}\right]\|f\|_{2} .
\end{aligned}
$$

This gives $i$ ii).

Note that these arguments do not use the self-adjointness of $A$. All what we need is that $-A$ generates a bounded holomorphic semigroup on $L^{2}$. Also one can reformulate $i i$ ) as follows

$$
\left\|\chi_{B(x, r)} u\right\|_{q} \leq \frac{C}{v(x, r)^{\gamma_{2, q}}}\left[\|u\|_{2}+r^{2 \beta}\left\|A^{\beta} u\right\|_{2}\right]
$$

for all $r>0$, all $x \in X$, and all $u \in D\left(A^{\beta}\right)$. The condition on $q$ becomes $\gamma_{2, q}<\frac{2 \beta}{N}$. The proof is the same.

One also obtains similar assertions by replacing the $L^{2}$ norm in the RHS by $L^{s}$ norm if $e^{-t A}$ is assumed to be bounded holomorphic on $L^{s}$.

We learned recently about somehow related results in [11] where the Gaussian estimate (3) of a heat kernel $p(t, x, y)$ is characterized in terms of inequalities of type

$$
\left\|f v_{r}^{\frac{1}{2}-\frac{1}{q}}\right\|_{q} \leq C\left(\|f\|_{2}+r^{2}\left\|A^{1 / 2} f\right\|_{2}\right) .
$$

Here $v_{r}(x):=v(x, r)$. For our latter purpose, these inequalities are not convenient because the heat kernel of $-\Delta+V$ with signed potential $V$ does not satisfy a Gaussian upper bound in general. We mentioned in the introduction that the semigroup $e^{-t(-\Delta+V)}$ may not act on all $L^{p}$ spaces. However, the inequality $i i$ ) in the previous proposition will allow us to prove $L^{p}-L^{q}$ off-diagonal estimates for $\chi_{B(x, r)} e^{-t(-\Delta+V)} \chi_{B(y, r)}$ for appropriate $p$ and $q$. 


\section{$2.2 \quad L^{p}-L^{q}$ off-diagonal estimate}

Let $A$ be as above and assume that there exists some $p_{1} \in(2, \infty]$ such that $\left(e^{-t A}\right)_{t>0}$ extends to a uniformly bounded semigroup on $L^{p}$ for all $p \in\left(p_{1}^{\prime}, p_{1}\right)$ where $p_{1}^{\prime}$ is the conjugate of $p_{1}$. Denote by $-A_{p}$ the corresponding generator of $\left(e^{-t A}\right)_{t>0}$ on $L^{p}$ (hence $A_{2}=A$ ). Suppose in addition that for $p \in\left(p_{1}^{\prime}, p_{1}\right)$, $\psi_{p}(u):=|u|^{p / 2} \operatorname{sign}(u) \in D\left(A^{1 / 2}\right)$ for all $u \in D\left(A_{p}\right) \cap L^{2}$ and

$$
\left\|A_{p} u\right\|_{p} \cdot\|u\|_{p}^{p-1} \geq C\left\|A^{1 / 2} \psi_{p}(u)\right\|_{2}^{2} .
$$

Here $|u|$ is the absolute value (or the modulus) of $u$ and sign is the classical sign function.

These hypothesis are always satisfied if the semigroup $e^{-t A}$ is sub-Markovian. Indeed, in this case, $\psi_{p}(u) \in D\left(A^{1 / 2}\right)$ for all $u \in D\left(A_{p}\right)$ with $p \in(1, \infty)$ and

$$
\left(A_{p} u,|u|^{p-1} \operatorname{sign}(u)\right) \geq 4 \frac{p-1}{p^{2}}\left\|A^{1 / 2} \psi_{p}(u)\right\|_{2}^{2}
$$

see [22] or Theorem 3.9 in [25].

Under these assumptions, we can obtain from the local $L^{2}-L^{q}$ estimate of Proposition 2.1 similar $L^{p}-L^{r}$ estimates where $r>p$, as explained in the following proposition.

Proposition 2.2. Let $A$ be a non-negative self-adjoint operator on $L^{2}$ such that its associated semigroup is uniformly bounded on $L^{p}$ for $p \in\left(p_{1}^{\prime}, p_{1}\right)$ and satisfies (4). Suppose also that ii) of Proposition 2.1 holds. Then

$$
\left\|\chi_{B(x, r)} e^{-s A}\right\|_{p-p q_{1}} \leq \frac{C}{v(x, r)^{\gamma_{p, p q_{1}}}}\left[\max \left(1, \frac{r}{\sqrt{s}}\right)\right]^{2 / p}
$$

for all $p \in\left[2, p_{1}\right)$ and all $q_{1} \in[1, \infty]$ if $N<2, q_{1} \in[1, \infty)$ if $N=2$ and $q_{1} \in\left[1, \frac{N}{N-2}\right)$ if $N>2$.

Proof: Let $u \in D\left(A_{p}\right) \cap L^{2}$. Since we assume $\psi_{p}(u) \in D\left(A^{1 / 2}\right)$ we can apply ii) of Proposition 2.1 to $\psi_{p}(u)$ (with the conditions there on $q$ ). This gives

$$
\left\|\chi_{B(x, r)}|u|^{p / 2}\right\|_{q} \leq \frac{C}{v(x, r)^{\gamma_{2, q}}}\left[\left|\left\|\left.u\right|^{p / 2}\right\|_{2}+r\left\|A^{1 / 2} \psi_{p}(u)\right\|_{2}\right] .\right.
$$

Using (4) we obtain

$$
\left\|\chi_{B(x, r)} u\right\|_{q p / 2}^{p / 2} \leq \frac{C}{v(x, r)^{\gamma_{2, q}}}\left[\|u\|_{p}^{p / 2}+r\left\|A_{p} u\right\|_{p}^{1 / 2} \cdot\|u\|_{p}^{(p-1) / 2}\right] .
$$

Take $f \in L^{2} \cap L^{p}$ and apply this inequality with $u=e^{-s A} f$. Since the semigroup is uniformly bounded on $L^{p}$ for all $p \in\left(p_{1}^{\prime}, p_{1}\right)$ and bounded 
analytic on $L^{2}$ it is well known by complex interpolation arguments that it is bounded analytic on $L^{p}$. Hence $\left\|A_{p} e^{-t A} f\right\|_{p} \leq C t^{-1}\|f\|_{p}$. Thus we obtain

$$
\left\|\chi_{B(x, r)} e^{-s A} f\right\|_{q p / 2}^{p / 2} \leq \frac{C}{v(x, r)^{\gamma_{2, q}}}\left[1+\frac{r}{\sqrt{s}}\right]\|f\|_{p}^{p / 2} .
$$

This is exactly (6) with $q_{1}:=\frac{q}{2} \in\left[1, \frac{N}{N-2}\right)$ if $N>2, q_{1} \in[1, \infty]$ for $N<2$, $q_{1} \in[1, \infty)$ if $N=2$.

Off-diagonal $L^{2}-L^{2}$ estimates ${ }^{2}$ are in general not difficult to prove by means of multiplicative perturbations. By such estimates we mean

$$
\left\|\chi_{F} e^{-t A} \chi_{E}\right\|_{2-2} \leq C e^{-c \rho^{2}(E, F) / t}
$$

for $E, F$ subsets of the metric space $(X, \rho)$ and $t>0$. Assume now that such estimates hold. Since we assume that the semigroup is uniformly bounded on $L^{p}$ for all $p \in\left(p_{1}^{\prime}, p_{1}\right)$, we obtain from the Riesz-Thorin interpolation theorem $L^{p}-L^{p}$ off-diagonal estimate

$$
\left\|\chi_{F} e^{-t A} \chi_{E}\right\|_{p-p} \leq C e^{-c \rho^{2}(E, F) / t}
$$

for all $p \in\left(p_{1}^{\prime}, p_{1}\right)$. The previous proposition implies $L^{p}-L^{p q}$ estimates

$$
\left\|\chi_{B(x, \sqrt{t})} e^{-t A} \chi_{B(y, \sqrt{t})}\right\|_{p-p q} \leq \frac{C}{v(x, \sqrt{t})^{\gamma_{p, p q}}}
$$

for all $p \in\left[2, p_{1}\right)$ and $q \in\left[1, q_{0}\right)$ with $q_{0}=\infty$ if $N \leq 2$ and $q_{0}:=\frac{N}{N-2}$ if $N>2$.

Using again the Riesz-Thorin interpolation theorem, (8) and (9) imply the following proposition

Proposition 2.3. Let $A$ be as in the previous proposition and assume that (7) holds. Then

$$
\left\|\chi_{B(x, \sqrt{t})} e^{-t A} \chi_{B(y, \sqrt{t})}\right\|_{r-s} \leq \frac{C}{v(x, \sqrt{t})^{\gamma_{r, s}}} e^{-c \rho^{2}(x, y) / t}
$$

for all $t>0, x, y \in X$, all $r \in\left[2, p_{1}\right), s \in\left[r, r q_{0}\right)$ where $q_{0}=\infty$ if $N \leq 2$ and $q_{0}=\frac{N}{N-2}$ if $N>2$.

By duality one has similar estimates for $\left\|\chi_{B(x, \sqrt{t})} e^{-t A} \chi_{B(y, \sqrt{t})} \mid\right\|_{s^{\prime}-r^{\prime}}$ with $v(y, \sqrt{t})$ instead of $v(x, \sqrt{t})$ in $(10)$. Again, $p^{\prime}$ denotes the conjugate exponent of $p$.

\footnotetext{
${ }^{2}$ also called Davies-Gaffney estimates
} 
Theorem 2.4. Let $(X, \rho, \mu)$ be a measured metric space satisfying the doubling condition (2) with some constant $N>0$. Let $A$ be as in the Proposition 2.2 and assume that (7) holds. Then the semigroup $\left(e^{-t A}\right)_{t>0}$ extends to a bounded analytic semigroup on $L^{p}$ for all $p \in\left(p_{0}^{\prime}, p_{0}\right)$ where $p_{0}:=p_{1} q_{0}$ and $q_{0}=\infty$ if $N \leq 2$ and $q_{0}=\frac{N}{N-2}$ if $N>2$.

Proof: From Proposition 2.2 we have

$$
\left\|\chi_{B(x, \sqrt{t})} e^{-t A} \chi_{A(x, \sqrt{t}, k)}\right\|_{r-s} \leq \frac{C}{v(x, \sqrt{t})^{\gamma_{r, s}}} .
$$

Here $A(x, \sqrt{t}, k)$ is the annulus

$$
A(x, \sqrt{t}, k):=B(x,(k+1) \sqrt{t}) \backslash B(x, k \sqrt{t}) .
$$

Interpolating again with (7) yields

$$
\left\|\chi_{B(x, \sqrt{t})} e^{-t A} \chi_{A(x, \sqrt{t}, k)}\right\|_{r-s} \leq \frac{C}{v(x, \sqrt{t})^{\gamma_{r, s}}} e^{-c k^{2}}
$$

for all $r \in\left[2, p_{1}\right)$ and all $s \in\left(2, p_{1} q_{0}\right)$, where $q_{0}=\infty$ if $N \leq 2$ and $q_{0}=\frac{N}{N-2}$ if $N>2$. Since the semigroup is bounded analytic on $L^{p}$ for $p \in\left(p_{1}^{\prime}, p_{1}\right)$ we can apply [7] Theorem 1.1 to deduce that the semigroup is bounded analytic on $L^{p}$ for all $p \in\left(2, p_{1} q_{0}\right)=\left(2, p_{0}\right)$. The case $p \in\left(p_{0}^{\prime}, 2\right)$ is obtained by duality. Note that we can also argue as in the proof of Proposition 2.9 below to obtain the boundedness of the semigroup on $L^{p}$ for $p$ as above. Analyticity on $L^{p}$ follows then from analyticity on $L^{2}$ by a classical complex interpolation argument.

We shall see later that the estimate of the previous proposition holds for $r$ and $s$ smaller than 2. Before proving this, we first need the following lemma.

Lemma 2.5. Assume that $X$ satisfies the doubling condition (2) for some constant $N>0$. For $a, r, s>0$, and $x, y \in X$, set

$$
J:=\int_{X}\left(1+\frac{\rho(B(x, r), B(z, r))}{r}\right)^{a} e^{-c^{\frac{\rho^{2}(B(x, r), B(z, r))}{s}}} d \mu(z) .
$$

Then

$$
J \leq C v(x, r)\left[\max \left(1, \frac{\sqrt{s}}{r}\right)\right]^{a+N} .
$$


Proof: By splitting the integral $J$ into two parts as $J_{1}+J_{2}:=\int_{B(x, 2 r)}+\int_{X \backslash B(x, 2 r)}$ we see that the first term is bounded by $C v(x, r)$ and then it remains to treat the second one. Therefore, we assume in the sequel that $\rho(x, z) \geq 2 r$. It is clear that

$$
\begin{aligned}
\left(1+\frac{\rho(B(x, r), B(z, r))}{r}\right)^{a} & =\left(1+\frac{\rho(B(x, r), B(z, r))}{\sqrt{s}} \frac{\sqrt{s}}{r}\right)^{a} \\
& \leq\left[\max \left(1, \frac{\sqrt{s}}{r}\right)\right]^{a}\left(1+\frac{\rho(B(x, r), B(z, r))}{\sqrt{s}}\right)^{a} .
\end{aligned}
$$

Using this, we obtain (with some constant $c>0$ slightly smaller than the one in $J$ )

$$
J_{2} \leq C\left[\max \left(1, \frac{\sqrt{s}}{r}\right)\right]^{a} \int_{X \backslash B(x, 2 r)} e^{-c \frac{\rho^{2}(B(x, r), B(z, r))}{s}} d \mu(z) .
$$

Assume that $\sqrt{s} \leq r$. Then

$$
J_{2} \leq C \int_{X \backslash B(x, 2 r)} e^{-c \frac{\rho^{2}(B(x, r), B(z, r))}{r^{2}}} d \mu(z),
$$

and since

$$
\rho^{2}(B(x, r), B(z, r)) \geq \rho(x, z)^{2}+4 r^{2}-4 r \rho(x, z)
$$

we end up with

$$
J_{2} \leq C \int_{X} e^{-c \frac{\rho^{2}(x, z)}{r^{2}}} d \mu(z)
$$

The standard argument which consists in decomposing $X$ into annuli $A(x, r, l)$ gives

$$
\begin{aligned}
\int_{X} e^{-c \frac{\rho^{2}(x, z)}{r^{2}}} d \mu(z) & \leq C \sum_{l=0}^{\infty} \int_{l r \leq \rho(x, z) \leq(l+1) r} e^{-c l^{2}} d \mu(z) \\
& \leq C \sum_{l=0}^{\infty} e^{-c l^{2}} v(x,(l+1) r) \leq C v(x, r)
\end{aligned}
$$

Assume now that $\sqrt{s}>r$. Then

$$
\begin{aligned}
J_{2} & \leq C\left(\frac{\sqrt{s}}{r}\right)^{a} \sum_{l=0}^{\infty} \int_{l \sqrt{s} \leq \rho(x, z) \leq(l+1) \sqrt{s}} e^{-c\left(\frac{\rho(x, z)}{\sqrt{s}}-\frac{2 r}{\sqrt{s}}\right)^{2}} d \mu(z) \\
& \leq C\left(\frac{\sqrt{s}}{r}\right)^{a} \sum_{l=0}^{\infty} e^{-c l^{2}}(l+1)^{N} v(x, \sqrt{s}) \\
& \leq C\left(\frac{\sqrt{s}}{r}\right)^{a+N} v(x, r) .
\end{aligned}
$$

This proves the lemma. 
Using the $L^{2}-L^{2}$ off diagonal estimate (7) and interpolation arguments we can deduce $L^{p}-L^{q}$ off diagonal estimates for $\chi_{B(x, r)} e^{-s A} \chi_{B(y, r)}$ for $p \in\left[2, p_{1}\right)$ and $q \in\left(p, p q_{0}\right)$ (see Proposition 2.3). Now we prove such estimates for all $p, q \in\left(p_{0}^{\prime}, p_{0}\right)$ such that $p \leq q$. More precisely, we have

Theorem 2.6. Let $X$ and $A$ be as in Theorem 2.4. Then for all $r, s>0$, and $p \in\left(p_{0}^{\prime}, p_{0}\right)$ and $q \in\left[p, p_{0}\right)$

$$
\left\|\chi_{B(x, r)} e^{-s A} \chi_{B(y, r)}\right\|_{p-q} \leq \frac{C}{v(x, r)^{\gamma_{p, q}}}\left(\max \left(\frac{r}{\sqrt{s}}, \frac{\sqrt{s}}{r}\right)\right)^{\beta} e^{-c \rho^{2}(B(x, r), B(y, r)) / s},
$$

where $C, c$, and $\beta$ are positive constants.

Proof: We first prove the theorem for $p=2$. That is

$$
\left\|\chi_{B(x, r)} e^{-s A} \chi_{B(y, r)}\right\|_{2-q} \leq \frac{C}{v(x, r)^{\gamma_{2, q}}}\left(\max \left(\frac{r}{\sqrt{s}}, \frac{\sqrt{s}}{r}\right)\right)^{\beta} e^{-c \rho^{2}(B(x, r), B(y, r)) / s},
$$

for $q \in\left[2, p_{0}\right)$.

From Theorem 2.4 and (7) we obtain by the Riesz-Thorin interpolation theorem the $L^{p}-L^{p}$ off-diagonal estimate

$$
\left\|\chi_{F} e^{-t A} \chi_{E}\right\|_{p-p} \leq C e^{-c \rho^{2}(E, F) / t}
$$

for all $p \in\left(p_{0}^{\prime}, p_{0}\right)$. Let us take a $p \in\left[2, p_{1}\right)$. Using once again interpolation we obtain from (6) and (15)

$$
\left\|\chi_{B(x, r)} e^{-s A} \chi_{B(y, r)}\right\|_{p-p u} \leq \frac{C}{v(x, r)^{\gamma_{p, p u}}}\left[\max \left(1, \frac{r}{\sqrt{s}}\right)\right]^{\theta} e^{-\frac{c \rho^{2}(B(x, r), B(y, r))}{s}}
$$

for all $p \in\left[2, p_{1}\right)$ and $u \in\left[1, q_{0}\right)$. Here $\theta$ is a positive constant depending on $p$ and $u$.

For $N \leq 2(16)$ is the $L^{2}-L^{s}$ off-diagonal estimate for all $s \in[2, \infty)$.

Suppose that $N>2$ and let us consider two cases. The first one is when $p_{1} \leq 2 q_{0}=\frac{2 N}{N-2}$ and the second one is when $2 q_{0}<p_{1}$.

Let us treat the first situation. Let $2<r_{1}<p_{1} \leq 2 q_{0}$ and $u<q_{0}\left(r_{1}\right.$ is close to $p_{1}$ and $u$ is close to $\left.q_{0}\right)$. We have from (16) $L^{2}-L^{r_{1}}$ and $L^{r_{1}}-L^{r_{1} u}$ off-diagonal estimates. We use a composition argument to obtain $L^{2}-L^{r_{1} u}$ estimate. For this, we need the following lemma (see [10] Lemma 3.4)

Lemma 2.7. Let $X$ be a metric measured space satisfying the doubling condition. If $R, S$ are two linear operators, we have

$$
\|R S\|_{p-q} \leq C \int_{X}\left\|R \chi_{B(z, r)}\right\|_{m-q}\left\|\chi_{B(z, s)} S\right\|_{p-m} v^{-1}(z, \inf (r, s)) d \mu(z)
$$


for all $r, s>0$ and all $1 \leq p \leq m \leq q \leq \infty$, where $C$ is independent of $R, S, r, s$.

Set $R:=\chi_{B(x, r)} e^{-\frac{s A}{2}}$ and $S:=e^{-\frac{s A}{2}} \chi_{B(y, r)}$. Then for all $u \in\left[1, q_{0}\right)$ (we are interested by $u$ close to $q_{0}$ ),

$$
\begin{aligned}
& \left\|\chi_{B(x, r)} e^{-s A} \chi_{B(y, r)}\right\|_{2-r_{1} u} \\
\leq & C \int_{X}\left\|\chi_{B(x, r)} e^{-\frac{s A}{2}} \chi_{B(z, r)}\right\|_{r_{1}-r_{1} u}\left\|\chi_{B(z, r)} e^{-\frac{s A}{2}} \chi_{B(y, r)}\right\|_{2-r_{1}} v^{-1}(z, r) d \mu(z) \\
\leq & \frac{C\left[\max \left(1, \frac{r}{\sqrt{s}}\right)\right]^{\theta}}{v(x, r)^{\gamma_{r_{1}, r_{1} u}}} \int_{X} \frac{e^{-\frac{c \rho^{2}(B(x, r), B(z, r))}{s}} e^{-\frac{c \rho^{2}(B(z, r), B(y, r))}{s}}}{v(z, r)^{\gamma_{2, r_{1}}+1}} d \mu(z) \\
\leq & \frac{C\left[\max \left(1, \frac{r}{\sqrt{s}}\right)\right]^{\theta}}{v(x, r)^{\gamma_{r_{1}, r_{1} u}}} e^{-\frac{c \rho^{2}(B(x, r), B(y, r))}{s}} \int_{X} \frac{e^{-\frac{c \rho^{2}(B(x, r), B(z, r))}{s}}}{v(z, r)^{\gamma_{2, r_{1}}+1}} d \mu(z)
\end{aligned}
$$

Using the fact that $B(x, r) \subset B(z, 3 r+\rho(B(x, r), B(z, r)))$ and the estimate (2), we deduce that

$$
\frac{1}{v(z, r)} \leq \frac{C}{v(x, r)}\left(3+\frac{\rho(B(x, r), B(z, r))}{r}\right)^{N} .
$$

Employing this in (17) yields

$$
\left\|\chi_{B(x, r)} e^{-s A} \chi_{B(y, r)}\right\|_{2-r_{1} u} \leq \frac{C\left[\max \left(1, \frac{r}{\sqrt{s}}\right)\right]^{\theta}}{v(x, r)^{\gamma_{2, r_{1} u}+1}} e^{-\frac{c \rho^{2}(B(x, r), B(y, r))}{s}} J,
$$

where

$$
J:=\int_{X}\left(3+\frac{\rho(B(x, r), B(z, r))}{r}\right)^{N\left(\gamma_{2, r_{1}}+1\right)} e^{-\frac{c \rho^{2}(B(x, r), B(z, r))}{s}} d \mu(z) .
$$

Now we use Lemma 2.5 and obtain $L^{2}-L^{r_{1} u}$ off-diagonal estimate for all $r_{1} \in\left[2, p_{1}\right)$ and $u \in\left[1, q_{0}\right)$. This is (14) for all $q \in\left[2, p_{0}\right)$.

Now we move to the second case, i.e. $2 q_{0}<p_{1}<p_{0}:=p_{1} q_{0}$. This is handled as before but with many decompositions. Indeed, if we choose $2 \leq p_{2}<\frac{2 N}{N-2}$, then we have the estimate (16) for $L^{2}-L^{p_{2}}$ and $L^{p_{2}}-L^{p_{3}}$ for $p_{2}<p_{3}=p_{2} u<p_{2} q_{0}$. The previous lemma gives the $L^{2}-L^{p_{3}}$ off-diagonal estimate. With several iterations we obtain $L^{2}-L^{r_{1}}$ off-diagonal estimate for some $r_{1} \in\left[p_{1}, p_{0}\right)$. Or, using (15) for $p_{0}-\varepsilon$ and the $L^{p_{1}-\varepsilon^{\prime}}-L^{p_{0}-\varepsilon}$ estimate obtained from (6) we obtain by interpolation $L^{p}-L^{p_{0}-\varepsilon}$ estimate as in (16) for all $p \in\left[p_{1}, p_{0}\right)$, in particular for $r_{1}$. Again, composing the $L^{2}-L^{r_{1}}$ and the 
$L^{r_{1}}-L^{p_{0}-\varepsilon}$ estimates yields a $L^{2}-L^{p_{0}-\varepsilon}$ estimate, so we obtain the desired estimate (14).

We apply the Riesz-Thorin interpolation theorem with (14) and (15) to obtain the estimate $(13)$ for $p \in\left[2, p_{0}\right)$ and $q \in\left[p, p_{0}\right)$. The case $p_{0}^{\prime}<p \leq$ $q \leq 2$ is obtained by duality of the case $2 \leq p \leq q<p_{0}$. By duality we have from (14) the $L^{p}-L^{2}$ off-diagonal estimate

$$
\left\|\chi_{B(x, r)} e^{-s A} \chi_{B(y, r)}\right\|_{p-2} \leq \frac{C}{v(y, r)^{\gamma_{p, 2}}}\left(\max \left(\frac{r}{\sqrt{s}}, \frac{\sqrt{s}}{r}\right)\right)^{\beta} e^{-c \rho^{2}(B(x, r), B(y, r)) / s}
$$

for all $p \in\left(p_{0}^{\prime}, 2\right]$. We argue as before by composing (20) and (14) for $q \in$ $\left[2, p_{0}\right)$ to end up with

$$
\left\|\chi_{B(x, r)} e^{-s A} \chi_{B(y, r)}\right\|_{p-q} \leq \frac{C}{v(y, r)^{\gamma_{p, 2}} v(x, r)^{\gamma_{2, q}}}\left(\max \left(\frac{r}{\sqrt{s}}, \frac{\sqrt{s}}{r}\right)\right)^{\beta} e^{-c^{\frac{\rho^{2}(B(x, r), B(y, r))}{s}}}
$$

where $p_{0}^{\prime}<p \leq 2 \leq q<p_{0}$. It remains to see that $v(y, r)$ in the RHS can be replaced by $v(x, r)$ (after changing the constants $c$ and $\beta$ ). Indeed, by the doubling property,

$$
\begin{aligned}
v(x, r) & \leq C v(y, r)\left[1+\frac{\rho(x, y)}{r}\right]^{N} \\
& \leq C v(y, r)\left[1+\frac{\rho(x, y)}{\sqrt{s}}\right]^{N}\left(\max \left(1, \frac{\sqrt{s}}{r}\right)\right)^{N} \\
& \leq C v(y, r)\left[1+\frac{\rho(B(x, r), B(y, r))}{\sqrt{s}}+\frac{2 r}{\sqrt{s}}\right]^{N}\left(\max \left(1, \frac{\sqrt{s}}{r}\right)\right)^{N} \\
& \leq C v(y, r)\left[1+\frac{\rho(B(x, r), B(y, r))}{\sqrt{s}}\right]^{N}\left(\max \left(\frac{r}{\sqrt{s}}, \frac{\sqrt{s}}{r}\right)\right)^{N} .
\end{aligned}
$$

Once we insert this in (21) we obtain (13) for $p \in\left(p_{0}^{\prime}, 2\right]$ and $q \in\left[2, p_{0}\right)$. Hence we have $(13)$ for all $p \in\left(p_{0}^{\prime}, p_{0}\right)$ and $q \in\left[p, p_{0}\right)$.

Corollary 2.8. (see also Proposition 2.1 in [10]) Let $X$ and $A$ be as in Theorem 2.4. For $p \in\left(p_{0}^{\prime}, p_{0}\right)$ and $q \in\left[p, p_{0}\right)$ we have for all $t>0$

$$
\begin{gathered}
\left\|\chi_{A(x, \sqrt{t}, k)} e^{-t A} \chi_{B(x, \sqrt{t})}\right\|_{p-q} \leq \frac{C}{v(x, \sqrt{t})^{\gamma_{p, q}}} e^{-c k^{2}} \\
\left\|\chi_{B(x, \sqrt{t})} e^{-t A} \chi_{A(x, \sqrt{t}, k)}\right\|_{p-q} \leq \frac{C}{v(x, \sqrt{t})^{\gamma_{p, q}}} e^{-c k^{2}} \\
\left\|\chi_{C_{j}(x, r)} e^{-s A} \chi_{B(x, r)}\right\|_{p-q} \leq \frac{C}{v(x, r)^{\gamma_{p, q}}}\left(\max \left(\frac{2^{j+1} r}{\sqrt{s}}, \frac{\sqrt{s}}{2^{j+1} r}\right)\right)^{\beta} e^{-c \frac{4^{j} r^{2}}{s}} .
\end{gathered}
$$


Proof: Using the obvious fact that

$$
\left\|\chi_{A(x, \sqrt{t}, k)} e^{-t A} \chi_{B(x, \sqrt{t})}\right\|_{p-q} \leq\left\|\chi_{B(x,(k+1) \sqrt{t})} e^{-t A} \chi_{B(x,(k+1) \sqrt{t})}\right\|_{p-q}
$$

and the previous theorem we obtain (for some constant $\beta$ )

$$
\left\|\chi_{A(x, \sqrt{t}, k)} e^{-t A} \chi_{B(x, \sqrt{t})}\right\|_{p-q} \leq \frac{C}{v(x, \sqrt{t})^{\gamma_{p, q}}}(k+1)^{\beta} .
$$

By (15) we know that

$$
\left\|\chi_{A(x, \sqrt{t}, k)} e^{-t A} \chi_{B(x, \sqrt{t})}\right\|_{p-p} \leq C e^{-c k^{2}}
$$

and we use interpolation to obtain (22). The arguments are similar for (23) and (24).

We finish this section by showing that $L^{p}-L^{q}$ off-diagonal estimates for $p \leq q$ imply that $\left(v(., \sqrt{t})^{\gamma_{p, q}} e^{-t A}\right)_{t>0}$ is $L^{p}-L^{q}$ bounded. What we prove here follows also from the results in [10] but we shall give details for the sake of completeness.

Proposition 2.9. Let $X$ and $A$ be as in Theorem 2.4. Then for all $p$ and $q$ as in the previous theorem,

$$
\left\|v(., \sqrt{t})^{\gamma_{p, q}} e^{-t A}\right\|_{p-q} \leq C .
$$

Proof: By Corollary 2.8 we have

$$
\left\|\chi_{B(x, \sqrt{t})} e^{-t A} \chi_{A(x, \sqrt{t}, k)}\right\|_{p-q} \leq \frac{C}{v(x, \sqrt{t})^{\gamma_{p, q}}} e^{-c k^{2}} .
$$

Using the fact that if $z \in B(x, \sqrt{t}), \quad v(z, \sqrt{t})$ is equivalent to $v(x, \sqrt{t})$ (by the doubling condition), we obtain

$$
\begin{aligned}
& \left\|\chi_{B(x, \sqrt{t})} v(., \sqrt{t})^{\gamma_{p, q}} e^{-t A} \chi_{A(x, \sqrt{t}, k)} f\right\|_{q}^{q} \\
= & \int_{X}\left|\chi_{B(x, \sqrt{t})}(z) v(z, \sqrt{t})^{\gamma_{p, q}} e^{-t A} \chi_{A(x, \sqrt{t}, k)}(z) f(z)\right|^{q} d \mu(z) \\
\leq & C e^{-c k^{2}}\|f\|_{p}^{q} .
\end{aligned}
$$

So we can write

$$
\begin{aligned}
& \left\|v(., \sqrt{t})^{\gamma_{p, q}} e^{-t A} f\right\|_{q}^{q} \\
= & \int_{X} v(x, \sqrt{t})^{-1} v(x, \sqrt{t})\left|v(x, \sqrt{t})^{\gamma_{p, q}} e^{-t A} f(x)\right|^{q} d \mu(x) \\
= & \int_{X} v(x, \sqrt{t})^{-1} \int_{M} \chi_{B(x, \sqrt{t})}(z) d \mu(z)\left|v(x, \sqrt{t})^{\gamma_{p, q}} e^{-t A} f(x)\right|^{q} d \mu(x) \\
\leq & C \int_{X} v(z, \sqrt{t})^{-1}\left\|\chi_{B(z, \sqrt{t})} v(., \sqrt{t})^{\gamma_{p, q}} e^{-t A} f\right\|_{q}^{q} d \mu(z) \\
= & C\left\|v(., \sqrt{t})^{-1 / q}\right\| \chi_{B(., \sqrt{t})} v(., \sqrt{t})^{\gamma_{p, q}} e^{-t A} f\left\|_{q}\right\|_{q}^{q} .
\end{aligned}
$$


Let us now estimate $v(z, \sqrt{t})^{-1 / q}\left\|\chi_{B(z, \sqrt{t})} v(., \sqrt{t})^{\gamma_{p, q}} e^{-t A} f\right\|_{q}$. By (26) we write

$$
\begin{aligned}
& v(z, \sqrt{t})^{-1 / q}\left\|\chi_{B(z, \sqrt{t})} v(., \sqrt{t})^{\gamma_{p, q}} e^{-t A} f\right\|_{q} \\
\leq & v(z, \sqrt{t})^{-1 / q} \sum_{k}\left\|\chi_{B(z, \sqrt{t})} v(., \sqrt{t})^{\gamma_{p, q}} e^{-t A} \chi_{A(z, \sqrt{t}, k)} f\right\|_{q} \\
\leq & C v(z, \sqrt{t})^{-1 / q} \sum_{k} e^{-c k^{2}}\left\|\chi_{A(z, \sqrt{t}, k)} f\right\|_{p} .
\end{aligned}
$$

Hence

$$
\begin{aligned}
& v(z, \sqrt{t})^{-1 / q}\left\|\chi_{B(z, \sqrt{t})} v(., \sqrt{t})^{\gamma_{p, q}} e^{-t A} f\right\|_{q} \\
\leq & C \sum_{k} e^{-c k^{2}} v(z,(k+1) \sqrt{t})^{-1 / q}\left\|\chi_{B(z,(k+1) \sqrt{t})} f\right\|_{p} .
\end{aligned}
$$

Inserting this in (27) we obtain

$$
\left\|v(., \sqrt{t})^{\gamma_{p, q}} e^{-t A} f\right\|_{q} \leq C \sum_{k} e^{-c k^{2}}\left\|v(.,(k+1) \sqrt{t})^{-\frac{1}{q}}\right\| \chi_{B(.,(k+1) \sqrt{t})} f\left\|_{p}\right\|_{q} .
$$

Then using the Minkowski inequality we estimate

$$
\begin{aligned}
& \left\|v(.,(k+1) \sqrt{t})^{-\frac{1}{q}}\right\| \chi_{B(.,(k+1) \sqrt{t})} f\left\|_{p}\right\|_{q} \\
\leq & {\left[\int_{X}|f(x)|^{p}\left(\int_{X} \chi_{B(y,(k+1) \sqrt{t})}(x) v(y,(k+1) \sqrt{t})^{-1} d \mu(y)\right)^{\frac{p}{q}} d \mu(x)\right]^{\frac{1}{p}} . }
\end{aligned}
$$

Thus,

$$
\left\|v(.,(k+1) \sqrt{t})^{-\frac{1}{q}}\right\| \chi_{B(.,(k+1) \sqrt{t})} f\left\|_{p}\right\|_{q} \leq\|f\|_{p},
$$

and we come to

$$
\left\|v(., \sqrt{t})^{\gamma_{p, q}} e^{-t A} f\right\|_{q} \leq C\|f\|_{p} \sum_{k} e^{-c k^{2}} \leq C\|f\|_{p} .
$$

Let us point out that all the results in this section hold with the same proofs if $A$ is considered on $L^{2}(\Omega)$ with $\Omega$ any open subset of $X$. The volume $v(x, r)$ appearing in the estimates is the volume of the ball $B(x, r)$ in $X$ not the volume of the ball in $\Omega$. When $(X, \rho, \mu)$ satisfies the doubling condition, $(\Omega, \rho, \mu)$ does not necessarily satisfy this propoerty. 


\section{The Schrödinger operator and the associ- ated Riesz transforms}

In this section we give applications of estimates proved in the previous one. We prove that these off-diagonal estimates are verified by the semigroup associated to the Schrödinger operator with signed strongly subcritical potential. Then we apply these estimates to prove the boundedness of the associated Riesz transforms on $L^{p}$ for $p \leq 2$, and their boundedness on $L^{p}$ for $p>2$ with additional conditions on the potential and on the manifold.

\subsection{The Schrödinger operator on $L^{2}$}

Let $M$ be a complete connected Riemannian manifold. Let $A$ be a Schrödinger operator $-\Delta+V$ on $L^{2}=L^{2}(M)$, where $-\Delta$ is the Laplace-Beltrami operator and the potential $V: M \rightarrow \mathbb{R}$ such that $V=V^{+}-V^{-}$(where $V^{+}$and $V^{-}$are the positive and negative parts of $V$, respectively). The operator is defined via the sesquilinear form method. We set

$$
\mathfrak{a}(u, v)=\int_{M} \nabla u \nabla v d \mu+\int_{M} V^{+} u v d \mu-\int_{M} V^{-} u v d \mu
$$

with domain

$$
D(\mathfrak{a})=\left\{u \in W^{1,2}(M), \int_{M} V^{+} u^{2} d \mu<\infty\right\} .
$$

Here we assume $V^{+} \in L_{\text {loc }}^{1}(M)$ and $V^{-}$satisfies (for all $u \in D(\mathfrak{a})$ ):

$$
\int_{M} V^{-} u^{2} d \mu \leq \alpha\left[\int_{M}|\nabla u|^{2} d \mu+\int_{M} V^{+} u^{2} d \mu\right]+\beta \int_{M} u^{2} d \mu
$$

where $\alpha \in(0,1)$ and $\beta \in \mathbb{R}$. By the well-known KLMN theorem (see for example [21] Chapter VI), the form $\mathfrak{a}$ is closed (and bounded from below). Its associated operator is $A=-\Delta+V$. If in addition $\beta \leq 0$, then $A$ is nonnegative.

Following [15], we take the following definition

Definition 3.1. We say that the negative part $V^{-}$is strongly subcritical if there exists an $0 \leq \alpha<1$ such that

$$
\int_{M} V^{-} u^{2} d \mu \leq \alpha\left[\int_{M}|\nabla u|^{2} d \mu+\int_{M} V^{+} u^{2} d \mu,\right]
$$

for all $u \in W^{1,2}(M)$ such that $\int_{M} V^{+} u^{2} d \mu<\infty$. 
Assume now that $V^{+} \in L_{l o c}^{1}$ and (29) holds. Set $A=-\Delta+V$ the Schrödinger operator defined above. Thus $A$ is a non-negative operator on $L^{2}(M)$. In addition

$$
(A u, u)=\int_{M}|\nabla u|^{2} d \mu+\int_{M} V^{+} u^{2} d \mu-\int_{M} V^{-} u^{2} d \mu \geq(1-\alpha) \int_{M}|\nabla u|^{2} d \mu .
$$

That is,

$$
\|\nabla u\|_{2}^{2} \leq \frac{1}{1-\alpha}\left\|A^{1 / 2} u\right\|_{2}^{2}
$$

Thus, the Riesz transform $\nabla A^{-1 / 2}$ is bounded on $L^{2}(M)$.

We also observe that, $V^{-}$is strongly subcritical means that $V^{-} \leq \alpha(-\Delta+$ $V^{+}$) which is equivalent to

$$
A=-\Delta+V^{+}-V^{-} \geq \frac{1-\alpha}{\alpha} V^{-} .
$$

Also the fact that $\alpha \in[0,1)$ gives

$$
A \geq(1-\alpha) V^{+} .
$$

Summing (31) and (32) gives

$$
A \geq \frac{1-\alpha}{2}\left(V^{+}+V^{-}\right)=\frac{1-\alpha}{2}|V| .
$$

This means that

$$
(A u, u) \geq \frac{1-\alpha}{2}(|V| u, u)=\frac{1-\alpha}{2} \int_{M}|V| u^{2} d \mu .
$$

That is,

$$
\left\||V|^{1 / 2} u\right\|_{2}^{2} \leq \frac{2}{1-\alpha}\left\|A^{1 / 2} u\right\|_{2}^{2}
$$

So we can conclude that

$$
\|\nabla u\|_{2}+\left\||V|^{1 / 2} u\right\|_{2} \leq C\left\|(-\Delta+V)^{1 / 2} u\right\|_{2}
$$

if $V^{-}$is strongly subcritical. Then by a duality argument we have

$$
\|\nabla u\|_{2}+\left\||V|^{1 / 2} u\right\|_{2} \approx\left\|(-\Delta+V)^{1 / 2} u\right\|_{2}
$$

if $V^{-}$is strongly subcritical.

As in [1] we obtain the following $L^{2}-L^{2}$ off-diagonal estimates (see also [14] for the first estimate). 
Proposition 3.2. Let $A$ be the Schrödinger operator with potential $V=$ $V^{+}-V^{-}$such that $V^{+} \in L_{l o c}^{1}$ and $V^{-}$satisfies (29). Then for all $E$ and $F$ subsests of $M$ and all $t>0$

(i) $\left\|\chi_{F} e^{-t A} \chi_{E}\right\|_{2-2} \leq e^{-c d^{2}(E, F) / t}$,

(ii) $\left\|\chi_{F} \sqrt{t} \nabla e^{-t A} \chi_{E}\right\|_{2-2} \leq C e^{-c d^{2}(E, F) / t}$,

(iii) $\left\|\chi_{F} \sqrt{t}|V|^{1 / 2} e^{-t A} \chi_{E}\right\|_{2-2} \leq C e^{-c d^{2}(E, F) / t}$.

\section{$3.2 \quad L^{p}$ Estimates}

In this subsection, we verify that the Schrödinger operator with potential $V$ such that $V^{-}$is strongly subcritical satisfies the assumptions of Subsection 2.2. Therefore its semigroup satisfies the $L^{p}-L^{q}$ off-diagonal estimates proved there.

Proposition 3.3. Let $A$ be a Schrödinger operator with $V^{+} \in L_{l o c}^{1}$ and suppose that $V^{-}$satisfies (29) with some constant $\alpha \in(0,1)$. Then the associated semigroup is uniformly bounded analytic on $L^{p}$ for all $p \in\left(p_{1}^{\prime}, p_{1}\right)$ where $p_{1}:=\frac{2}{1-\sqrt{1-\alpha}}$.

This result follows from [22], Theorem 3.2. The semigroup is even a contraction one on $L^{p}$ for $p \in\left(p_{1}^{\prime}, p_{1}\right)$. In order to understand this, note that for a smooth function $u$,

$$
\left(-\Delta u,|u|^{p-1} \operatorname{sign}(u)\right)=\frac{4(p-1)}{p^{2}}\left(\nabla\left(|u|^{p / 2} \operatorname{sign}(u)\right), \nabla\left(|u|^{p / 2} \operatorname{sign}(u)\right)\right) .
$$

Therefore,

$$
\begin{aligned}
\left(A u,|u|^{p-1} \operatorname{sign}(u)\right)= & \frac{4(p-1)}{p^{2}}\left(\nabla\left(|u|^{p / 2} \operatorname{sign}(u)\right), \nabla\left(|u|^{p / 2} \operatorname{sign}(u)\right)\right) \\
& +\left(V^{+}|u|^{p / 2},|u|^{p / 2}\right)-\left(V^{-}|u|^{p / 2},|u|^{p / 2}\right) \\
\geq & \left(\frac{4(p-1)}{p^{2}}-\alpha\right)\left[\left(\nabla\left(|u|^{p / 2} \operatorname{sign}(u)\right), \nabla\left(|u|^{p / 2} \operatorname{sign}(u)\right)\right] .\right.
\end{aligned}
$$

This extends by density arguments to all $u$ in an appropriate domain of $A$ as an operator in $L^{p}$. This explains why $A$ is a dissipative operator on $L^{p}$ for $p$ such that $\frac{4(p-1)}{p^{2}}-\alpha \geq 0$, i.e., for $p \in\left(p_{1}^{\prime}, p_{1}\right)$ with $p_{1}=\frac{2}{1-\sqrt{1-\alpha}}$. For more details, see [22].

The previous inequalities show also that

$$
\left(A u,|u|^{p-1} \operatorname{sign}(u)\right) \geq C\left(A^{1 / 2}|u|^{p / 2} \operatorname{sign}(u), A^{1 / 2}|u|^{p / 2} \operatorname{sign}(u)\right),
$$

which gives (4). 
Theorem 3.4. Assume that the manifold $M$ satisfies the doubling condition (2) and the heat kernel of the Laplace-Beltrami operator satisfies the Gaussian upper bound (3). Let $A$ be the Schrödinger operator with $V^{+} \in L_{l o c}^{1}$ and $V^{-}$ strongly subcritical. We have following properties.

1) The semigroup $\left(e^{-t A}\right)$ extends to a bounded analytic semigroup on $L^{p}$ for all $p \in\left(p_{0}^{\prime}, p_{0}\right)$. Here $p_{0}=\infty$ if $N \leq 2$ and $p_{0}=\left(\frac{2}{1-\sqrt{1-\alpha}}\right) \frac{N}{N-2}$ if $N>2$.

2) For all $r, s>0, x, y \in M$ and all $p \in\left(p_{0}^{\prime}, p_{0}\right), q \in\left[p, p_{0}\right)$

$$
\begin{aligned}
& \left\|\chi_{B(x, r)} e^{-s A} \chi_{B(y, r)}\right\|_{p-q} \leq \frac{C}{v(x, r)^{\gamma_{p, q}}}\left(\max \left(\frac{r}{\sqrt{s}}, \frac{\sqrt{s}}{r}\right)\right)^{\beta} e^{-c d^{2}(B(x, r), B(y, r)) / s}, \\
& \left\|\chi_{A(x, \sqrt{t}, k)} e^{-t A} \chi_{B(x, \sqrt{t})}\right\|_{p-q} \leq \frac{C}{v(x, \sqrt{t})^{\gamma_{p, q}}} e^{-c k^{2}} \\
& \left\|\chi_{B(x, \sqrt{t})} e^{-t A} \chi_{A(x, \sqrt{t}, k)}\right\|_{p-q} \leq \frac{C}{v(x, \sqrt{t})^{\gamma_{p, q}}} e^{-c k^{2}}, \\
& \left\|v(., \sqrt{t})^{\gamma_{p, q}} e^{-t A}\right\|_{p-q} \leq C . \\
& \left\|\chi_{C_{j}(x, r)} e^{-s A} \chi_{B(x, r)}\right\|_{p-q} \leq \frac{C}{v(x, r)^{\gamma_{p, q}}}\left(\max \left(\frac{2^{j+1} r}{\sqrt{s}}, \frac{\sqrt{s}}{2^{j+1} r}\right)\right)^{\beta} e^{-c \frac{4^{j} r^{2}}{s}} .
\end{aligned}
$$

Proof: By the previous proposition and (35) we can apply the results of the previous section (Theorems 2.4, 2.6, Proposition 2.9 and Corollary 2.8). The missing thing at this stage is property ii) of Proposition 2.1 for $A$. Since the heat kernel of $-\Delta$ satisfies the Gaussian bound (3), it is easily seen that

$$
\left\|\chi_{B(x, \sqrt{t})} e^{-s(-\Delta)}\right\|_{2-\infty} \leq \frac{C}{v(x, \sqrt{t})^{1 / 2}}\left(\frac{t}{s}\right)^{\frac{N}{4}}
$$

for all $0<s \leq t$. Thus, estimate i) of Proposition 2.1 holds for $-\Delta$ and this implies $i$ ) for $-\Delta$. As explained before, the Riesz transform of $A$ is bounded on $L^{2}$, hence

$$
\left\|(-\Delta)^{1 / 2} f\right\|_{2}=\|\nabla f\|_{2} \leq C\left\|A^{1 / 2}\right\|_{2} .
$$

This implies that property ii) of Proposition 2.1 holds for $A$.

\section{3 $\quad L^{p}-L^{2}$ off-diagonal estimates of $\nabla e^{-s A}$ and $|V|^{1 / 2} e^{-s A}$}

In this subsection, we combine the $L^{2}-L^{p}$ off-diagonal estimates of the semigroup with the $L^{2}-L^{2}$ off-diagonal estimates of Proposition 3.2 to obtain the following result.

Theorem 3.5. Let $M$ be a complete Riemannian manifold where (2) and (3) are satisfied. Let $A=-\Delta+V$ be a Schrödinger operator with $V^{+} \in L_{l o c}^{1}$ and $V^{-}$strongly subcritical. Denote by $T_{s}$ either $\sqrt{s} \nabla e^{-s A}$ or $\sqrt{s}|V|^{1 / 2} e^{-s A}$. 
Then $T_{s}$ satisfies the following $L^{p}-L^{2}$ off-diagonal estimates for all $p \in$ $\left(p_{0}^{\prime}, 2\right]$, where $p_{0}^{\prime}=1$ if $N \leq 2$, and $p_{0}^{\prime}=\left(\frac{2 N}{(N-2)(1-\sqrt{1-\alpha})}\right)^{\prime}$ if $N>2$.

$$
\begin{aligned}
\left\|\chi_{B(x, r)} T_{s} \chi_{B(y, r)}\right\|_{p-2} & \leq \frac{C g(r, s)}{v(y, r)^{\gamma_{p, 2}}} e^{-c \frac{d^{2}(B(x, r), B(y, r))}{s}} \\
\left\|\chi_{A(x, r, l)} T_{s} \chi_{B(x, r)}\right\|_{p-2} & \leq \frac{C g(r, s) l^{\beta}}{v(x, r)^{\gamma_{p, 2}}} e^{-c \frac{l^{2} r^{2}}{s}} \\
\left\|\chi_{C_{j}(x, r)} T_{s} \chi_{B(x, r)}\right\|_{p-2} & \leq \frac{C g(r, s) 2^{j \beta}}{v(x, r)^{\gamma_{p, 2}}} e^{-c \frac{4^{j} r^{2}}{s}}
\end{aligned}
$$

where $g(r, s):=\left(\max \left(\frac{r}{\sqrt{s}}, \frac{\sqrt{s}}{r}\right)\right)^{\beta}$ for some positive constant $\beta$. We recall that $A(x, r, k):=B(x,(k+1) r) \backslash B(x, k r), C_{1}(x, r):=B(x, 4 r), C_{j}(x, r):=$ $B\left(x, 2^{j+1} r\right) \backslash B\left(x, 2^{j} r\right)$ for $j \geq 2$.

Proof: Let us take $T_{s}=\sqrt{s} \nabla e^{-s A}$. The proof is the same for $T_{s}=\sqrt{s}|V|^{1 / 2} e^{-s A}$.

First, we prove (36).

By Theorem 3.4,

$$
\left\|\chi_{B(z, r)} e^{-s A} \chi_{B(y, r)}\right\|_{p-2} \leq \frac{C g(r, s)}{v(z, r)^{\gamma_{p, 2}}} e^{-\frac{c d^{2}(B(z, r), B(y, r))}{s}}
$$

for all $p \in\left(p_{0}^{\prime}, 2\right]$. We also have the $L^{2}-L^{2}$ off-diagonal estimate (ii) of Proposition 3.2, which gives

$$
\left\|\chi_{B(x, r)} \sqrt{s} \nabla e^{-s A} \chi_{B(z, r)}\right\|_{2-2} \leq C e^{-c d^{2}(B(x, r), B(z, r)) / s} .
$$

Now, as before, we use Lemma 2.7 and write

$$
\begin{aligned}
& \left\|\chi_{B(x, r)} \sqrt{s} \nabla e^{-s A} \chi_{B(y, r)}\right\|_{p-2} \\
\leq & C \int\left\|\chi_{B(x, r)} \sqrt{s} \nabla e^{-\frac{s A}{2}} \chi_{B(z, r)}\right\|_{2-2}\left\|\chi_{B(z, r)} e^{-\frac{s A}{2}} \chi_{B(y, r)}\right\|_{p-2} v^{-1}(z, r) d \mu(z) .
\end{aligned}
$$

We employ (39) , (40), and (18) to obtain

$$
\left\|\chi_{B(x, r)} \sqrt{s} \nabla e^{-s A} \chi_{B(y, r)}\right\|_{p-2} \leq \frac{C g(r, s)}{v(y, r)^{\gamma_{p, 2}+1}} e^{-\frac{c d^{2}(B(x, r), B(y, r))}{s}} I,
$$

where

$$
I:=\int_{M}\left(1+\frac{d(B(y, r), B(z, r))}{r}\right)^{N\left(\gamma_{p, 2}+1\right)} e^{-\frac{c d^{2}(B(y, r), B(z, r))}{s}} d \mu(z) .
$$

Now we use Lemma 2.5 and obtain (36). 
Now, we check (37).

By (36), we have

$$
\begin{aligned}
\left\|\chi_{A(x, r, l)} T_{s} \chi_{B(x, r)}\right\|_{p-2} & \leq\left\|\chi_{B(x,(l+1) r)} T_{s} \chi_{B(x,(l+1) r)}\right\|_{p-2} \\
& \leq \frac{C g((l+1) r, s)}{v(x, r)^{\gamma_{p, 2}}} \\
& \leq \frac{C g(r, s) l^{\beta}}{v(x, r)^{\gamma_{p, 2}}}
\end{aligned}
$$

By Proposition 3.2

$$
\left\|\chi_{A(x, r, l)} T_{s} \chi_{B(x, r)}\right\|_{2-2} \leq C e^{-c \frac{l^{2} r^{2}}{s}} .
$$

Therefore, (37) holds by applying the Riesz-Thorin interpolation theorem.

The latter arguments work also for (38).

\subsection{Boundedness of Riesz transforms $\nabla A^{-1 / 2}$ and of $|V|^{1 / 2} A^{-1 / 2}$ on $L^{p}, p<2$}

Having the main tools for the Schrödinger operator $A=-\Delta+V^{+}-V^{-}$, we can prove the boundedness of $\nabla A^{-1 / 2}$ and of $|V|^{1 / 2} A^{-1 / 2}$ on $L^{p}$ for all $p \in(1,2]$ if $N \leq 2$, and for all $p \in\left(p_{0}^{\prime}, 2\right]$ if $N>2$. More precisely,

Theorem 3.6. Let $M$ be the Riemannian manifold with assumptions (2) and (3). Let $A$ be the Schrödinger operator with signed potential $V$ such that $V^{+} \in L_{l o c}^{1}$ and $V^{-}$satisfies (29). Then the associated Riesz transforms $\nabla A^{-1 / 2}$ and the operator $|V|^{1 / 2} A^{-1 / 2}$ are bounded on $L^{p}$ for all $p \in(1,2]$ if $N \leq 2$ and for all $p \in\left(p_{0}^{\prime}, 2\right]$ if $N>2$ where $p_{0}^{\prime}:=\left(\frac{2 N}{(N-2)(1-\sqrt{1-\alpha})}\right)^{\prime}$.

Proof: To prove this result, we prove that $\nabla A^{-1 / 2}$ and $|V|^{1 / 2} A^{-1 / 2}$ are of weak type $(p, p)$, then using the boundedness on $L^{2}$ and the Marcinkiewicz interpolation theorem we deduce the boundedness on $L^{p}$ for the suitable range of $p$. To prove that $\nabla A^{-1 / 2}$ and $|V|^{1 / 2} A^{-1 / 2}$ are of weak type $(p, p)$ we apply the following theorem from [8]. The formulation here is taken from [2].

Theorem 3.7. Let $p \in[1,2)$. Suppose that $T$ is sublinear operator of strong type $(2,2)$, and let $\left(A_{r}\right)_{r>0}$ be a family of linear operators acting on $L^{2}$. Assume that for $j \geq 2$ and every ball $B=B(x, r)$,

$$
\left(\frac{1}{v\left(x, 2^{j+1} r\right)} \int_{C_{j}(x, r)}\left|T\left(I-A_{r}\right) f\right|^{2}\right)^{1 / 2} \leq g(j)\left(\frac{1}{v(x, r)} \int_{B}|f|^{p}\right)^{1 / p},
$$


and for $j \geq 1$

$$
\left(\frac{1}{v\left(x, 2^{j+1} r\right)} \int_{C_{j}(x, r)}\left|A_{r} f\right|^{2}\right)^{1 / 2} \leq g(j)\left(\frac{1}{v(x, r)} \int_{B}|f|^{p}\right)^{1 / p},
$$

for all $f$ supported in $B$. If $\Sigma:=\sum g(j) 2^{N j}<\infty$, then $T$ is of weak type $(p, p)$, with a bound depending only on the strong type $(2,2)$ bound of $T, p$, and $\Sigma$.

Set $T:=\nabla A^{-1 / 2}$ (the case $T=|V|^{1 / 2} A^{-1 / 2}$ is treated in a similar way). We prove assumptions (43) and (44) with $A_{r}=I-\left(I-e^{-r^{2} A}\right)^{m}$ for some integer $m$ large enough. The following arguments are very similar to those in [2] Theorem 4.2 and [1] where some elliptic and Schrödinger operators are treated on the Euclidean space.

We prove (44) by using the estimate of Theorem 3.4. For $f$ supported in a ball $B=B(x, r)$,

$$
\begin{aligned}
& \frac{1}{v\left(x, 2^{j+1} r\right)^{1 / 2}}\left\|A_{r} f\right\|_{L^{2}\left(C_{j}(x, r)\right)} \\
= & \frac{1}{v\left(x, 2^{j+1} r\right)^{1 / 2}}\left\|\sum_{k=1}^{m}\left(\begin{array}{c}
m \\
k
\end{array}\right)(-1)^{k+1} e^{-k r^{2} A} f\right\|_{L^{2}\left(C_{j}(x, r)\right)} \\
\leq & \frac{1}{v(x, r)^{1 / 2}} \sum_{k=1}^{m}\left(\begin{array}{c}
m \\
k
\end{array}\right)\left\|e^{-k r^{2} A} f\right\|_{L^{2}\left(C_{j}(x, r)\right)} \\
\leq & \frac{C}{v(x, r)^{1 / 2}} \frac{1}{v(x, r)^{\gamma_{p, 2}}} 2^{j+1} e^{-c 4^{j}}\|f\|_{L^{p}(B)} \\
\leq & C e^{-c 4^{j}}\left(\frac{1}{v(x, r)} \int_{B}|f|^{p}\right)^{1 / p}
\end{aligned}
$$

From this it is clear that (44) holds with $g(j)=C e^{-c 4^{j}}$.

It remains to check the assumption (43). Fix $f \in L^{2}$ supported in a ball $B$. We know that

$$
\nabla A^{-1 / 2} f=C \int_{0}^{\infty} \nabla e^{-t A} f \frac{d t}{\sqrt{t}}
$$

and hence

$$
\begin{aligned}
\nabla A^{-1 / 2}\left(I-e^{-r^{2} A}\right)^{m} f & =C \int_{0}^{\infty} \nabla e^{-t A}\left(I-e^{-r^{2} A}\right)^{m} f \frac{d t}{\sqrt{t}} \\
& =C \int_{0}^{\infty} g_{r^{2}}(t) \nabla e^{-t A} f d t
\end{aligned}
$$

where

$$
g_{r^{2}}(t)=\sum_{k=0}^{m}\left(\begin{array}{c}
m \\
k
\end{array}\right)(-1)^{k} \frac{\chi_{\left(t-k r^{2}>0\right)}}{\sqrt{t-k r^{2}}}
$$


By Theorem 3.5

$$
\begin{aligned}
\left\|\chi_{C_{j}(x, r)} \nabla A^{-1 / 2}\left(I-A_{r}\right) \chi_{B}\right\|_{p-2} & \leq \frac{C 2^{j \beta}}{v(x, r)^{\gamma_{p, 2}}} \int_{0}^{\infty}\left|g_{r^{2}}(t) g(r, t)\right| e^{-c \frac{4^{j} r^{2}}{t}} \frac{d t}{\sqrt{t}} \\
& =\frac{C 2^{j \beta}}{v(x, r)^{\gamma_{p, 2}}}\left(I_{1}+I_{2}\right),
\end{aligned}
$$

where

$$
I_{1}:=\int_{0}^{(m+1) r^{2}}\left|g_{r^{2}}(t) g(r, t)\right| e^{-\frac{4^{j} r^{2}}{t}} \frac{d t}{\sqrt{t}}
$$

and

$$
I_{2}:=\int_{(m+1) r^{2}}^{\infty}\left|g_{r^{2}}(t) g(r, t)\right| e^{-c \frac{4^{j} r^{2}}{t}} \frac{d t}{\sqrt{t}} .
$$

We observe that (see [2] p. 27)

$$
\left|g_{r^{2}}(t)\right| \leq \frac{C}{\sqrt{t-k r^{2}}} \quad \text { if } \quad k r^{2}<t \leq(k+1) r^{2} \leq(m+1) r^{2}
$$

and

$$
\left|g_{r^{2}}(t)\right| \leq C r^{2 m} t^{-m-1 / 2} \quad \text { if } \quad t>(m+1) r^{2} .
$$

Using this we have

$$
\begin{aligned}
I_{1}= & \int_{0}^{r^{2}}\left(\frac{r}{\sqrt{t}}\right)^{\beta} e^{-c \frac{4^{j} r^{2}}{t}} \frac{d t}{t}+\sum_{k=1}^{m} \int_{k r^{2}}^{(k+1) r^{2}}\left|g_{r^{2}}(t) g(r, t)\right| e^{-c \frac{4^{j} r^{2}}{t}} \frac{d t}{\sqrt{t}} \\
\leq & 2^{-j \beta} \int_{0}^{4^{-j}} e^{-c \frac{1}{u}} \frac{d u}{u^{1+\beta / 2}} \\
& +C r^{-1} e^{-c^{\prime} 4^{j}} \sum_{k=1}^{m} \frac{1}{\sqrt{k}} \int_{k r^{2}}^{(k+1) r^{2}} \max \left(\frac{r}{\sqrt{t}}, \frac{\sqrt{t}}{r}\right)^{\beta}\left(t-k r^{2}\right)^{-1 / 2} d t \\
\leq & C e^{-c^{\prime} 4^{j}} .
\end{aligned}
$$

On the other hand, the obvious bound $\lambda^{\alpha} e^{-\lambda} \leq C$ for any positive $\alpha$ gives (here we choose $\alpha=m$ large enough)

$$
\begin{aligned}
I_{2} & \leq C r^{2 m} \int_{(m+1) r^{2}}^{\infty} t^{-m-1} \max \left(\frac{r}{\sqrt{t}}, \frac{\sqrt{t}}{r}\right)^{\beta} e^{-c \frac{4^{j} r^{2}}{t}} d t \\
& \leq C 4^{-j m} r^{2 m-2 m-\beta} \int_{(m+1) r^{2}}^{\infty} t^{-m-1+\frac{\beta}{2}+m} d t \\
& =C^{\prime} 4^{-j m} .
\end{aligned}
$$

We obtain from these estimates

$$
\left\|\chi_{C_{j}(x, r)} \nabla A^{-1 / 2}\left(I-A_{r}\right) \chi_{B}\right\|_{p-2} \leq \frac{C}{v(x, r)^{\gamma_{p, 2}}} 2^{j \beta}\left(e^{-c^{\prime} 4^{j}}+4^{-j m}\right) .
$$

This shows (43) and proves the theorem. 
Remarks: 1) As explained in [1] in the Euclidean case, the value $p_{0}^{\prime}$ is optimal in the sense that the Riesz transform $\nabla A^{-1 / 2}$ is bounded on $L^{p}$ for $p \in\left(p_{0}^{\prime}, 2\right]$ and could be unbounded on $L^{p}$ for $p<p_{0}^{\prime}$. The reason is that this value is also optimal for the existence of the semigroup $e^{-t A}$ on $L^{p}$ for $p \in\left(p_{0}^{\prime}, p_{0}\right)$.

2) Suppose that $N>2$ and $V^{-}$satisfies (29). Suppose in addition that $V^{-} \in K_{\infty}(M)$, that is, for any $\varepsilon>0$ there exists a compact set $K \subset M$ and $\delta>0$ such that

$$
\sup _{x \in M} \int_{K^{c}} G(x, y)\left|V^{-}(y)\right| d \mu(y) \leq \varepsilon
$$

where $G(x, y)$ is the Green function, $K^{c}:=M \backslash K$, and for all measurable sets $B \subset K$ with $\mu(B)<\delta$,

$$
\sup _{x \in M} \int_{B} G(x, y)\left|V^{-}(y)\right| d \mu(y) \leq \varepsilon .
$$

Suppose also that $L^{2}$-Poincaré inequalities hold. Then using the results in [29], it follows that the heat kernel of $A$ has a Gaussian upper bound. Therefore, Theorem 5 in [27] shows that $\nabla A^{-1 / 2}$ and $|V|^{1 / 2} A^{-1 / 2}$ are of weak type $(1,1)$ and they are bounded on $L^{p}$ for all $p \in(1,2]$.

\subsection{Boundedness of $V A^{-1}$ and $(-\Delta) A^{-1}$ on $L^{p}$}

Let us first recall again the notation $p_{0}=\left(\frac{2}{1-\sqrt{1-\alpha}}\right) \frac{N}{N-2}$ if $N>2$ and $p_{0}=\infty$ otherwise.

Proposition 3.8. Let $M$ be a complete Riemannian manifold for which (2) and (3) hold. Let $A$ be the Schrödinger operator with signed potential $V$ such that $V^{+} \in L_{l o c}^{1}$ and $V^{-}$satisfying (29). Assume that, for some $r_{1}, r_{2}>2$

$$
\int_{0}^{1}\left\|\frac{V}{v(., \sqrt{s})^{\frac{1}{r_{1}}}}\right\|_{r_{1}} d s<\infty \text { and } \int_{1}^{\infty}\left\|\frac{V}{v(., \sqrt{s})^{\frac{1}{r_{2}}}}\right\|_{r_{2}} d s<\infty .
$$

Set $r:=\inf \left(r_{1}, r_{2}\right)$. Then $V A^{-1}$ and $(-\Delta) A^{-1}$ are bounded on $L^{p}$ for all $p \in\left(p_{0}^{\prime}, \frac{p_{0} r}{r+p_{0}}\right)$ if $N>2$ and $p \in(1, r)$ if $N \leq 2$.

Proof: Let us write ${ }^{3}$

$$
A^{-1}=\int_{0}^{\infty} e^{-s A} d s
$$

\footnotetext{
${ }^{3}$ we use $A^{-1}$ for simplicity. In principle we should replace in this formula $A$ by $\varepsilon I+A$, the estimates we prove below are uniform with respect to $\varepsilon$, we then let $\varepsilon \rightarrow 0$.
} 
By Hölder's inequality,

$$
\begin{aligned}
\left\|V A^{-1}\right\|_{p-p} & \leq \int_{0}^{\infty}\left\|V e^{-s A}\right\|_{p-p} d s \\
& \leq C \int_{0}^{1}\left\|\frac{V}{v(., \sqrt{s})^{\frac{1}{r_{1}}}}\right\|_{r_{1}}\left\|v(., \sqrt{s})^{\frac{1}{r_{1}}} e^{-s A}\right\|_{p-q_{1}} d s \\
& +C \int_{1}^{\infty}\left\|\frac{V}{v(., \sqrt{s})^{\frac{1}{r_{2}}}}\right\|_{r_{2}}\left\|v(., \sqrt{s})^{\frac{1}{r_{2}}} e^{-s A}\right\|_{p-q_{2}} d s
\end{aligned}
$$

for all $p \in\left(p_{0}^{\prime}, p_{0}\right)$ such that $\frac{1}{p}-\frac{1}{q_{1}}=\frac{1}{r_{1}}$ and $\frac{1}{p}-\frac{1}{q_{2}}=\frac{1}{r_{2}}$. Proposition 2.9 applied to $A$ gives (note that $\gamma_{p, q_{1}}=\frac{1}{r_{1}}$ and $\gamma_{p, q_{2}}=\frac{1}{r_{2}}$ )

$$
\left\|v(., \sqrt{s})^{\gamma_{p, q}} e^{-s A}\right\|_{p-q} \leq C
$$

for $p_{0}^{\prime}<p \leq q<p_{0}$. We choose $q_{1}$ and $q_{2}$ close to $p_{0}$ and using (45), we deduce

$$
\begin{aligned}
\left\|V A^{-1}\right\|_{p-p} & \leq C \int_{0}^{1}\left\|\frac{V}{v(., \sqrt{s})^{\frac{1}{r_{1}}}}\right\|_{r_{1}} d s+C \int_{1}^{\infty}\left\|\frac{V}{v(., \sqrt{s})^{\frac{1}{r_{2}}}}\right\|_{r_{2}} d s \\
& \leq C
\end{aligned}
$$

for all $p \in\left(p_{0}^{\prime}, \frac{r p_{0}}{r+p_{0}}\right)$. Finally, for $u \in L^{2} \cap L^{p}$

$$
\begin{aligned}
\left\|(-\Delta) A^{-1} u\right\|_{p} & =\left\|(-\Delta+V-V) A^{-1} u\right\|_{p} \\
& \leq\|u\|_{p}+\left\|V A^{-1} u\right\|_{p} \\
& \leq C\|u\|_{p} .
\end{aligned}
$$

Remarks. 1) If $L^{2}$-Poincaré inequality holds and $V^{-}$is such that the heat kernel of $A$ has a Gaussian upper bound (see the remark at the end of the previous section). Then (46) holds for all $p \leq q \in(1, \infty)$. This means that there will be no restriction on $q_{1}$ and $q_{2}$ in the previous proof. With the same assumptions as in the previous proposition, we end up with $V A^{-1}$ and $(-\Delta) A^{-1}$ bounded on $L^{p}$ for all $p \in(1, r)$.

2) Assume that $v(x, t) \approx t^{N}$ for all $x \in M, t>0$, with $N>2$. Then (45) means that $V \in L^{\frac{N}{2}-\varepsilon} \cap L^{\frac{N}{2}+\varepsilon}$ for some $\varepsilon>0$. Assume also that $L^{2}$-Poincaré inequality holds. The conclusion is then $V A^{-1}$ and $(-\Delta) A^{-1}$ are bounded on $L^{p}$ for $p \in\left(p_{0}^{\prime}, \frac{p_{0} N}{2 p_{0}+N}\right)$. We know in this case (see [1] Corollary 4.1 and its proof) that the heat kernel of $A=-\Delta+V$ satisfies the Gaussian upper bound (3). This means that $p_{0}=\infty$ and we obtain boundedness of $V A^{-1}$ and $(-\Delta) A^{-1}$ on $L^{p}$ for $p \in(1, N / 2)$. For related results with conditions that $V^{+}$in a reverse Hölder class, see [28], [3] and [6]. 


\subsection{Boundedness of $\nabla A^{-1 / 2}$ and $|V|^{1 / 2} A^{-1 / 2}$ on $L^{p}$ for $p>2$}

In this section we study boundedness of the Riesz transform of $A$ on $L^{p}$ for $p>2$.

Theorem 3.9. Let $M$ be a complete Riemannian manifold with (2) and (3). Let $A$ be the Schrödinger operator with signed potential $V$ such that $V^{+} \in L_{l o c}^{1}$ and $V^{-}$satisfying (29). Assume that, for some $r_{1}, r_{2}>2$

$$
\int_{0}^{1}\left\|\frac{|V|^{1 / 2}}{v(., \sqrt{s})^{\frac{1}{r_{1}}}}\right\|_{r_{1}} \frac{d s}{\sqrt{s}}<\infty \quad \text { and } \quad \int_{1}^{\infty}\left\|\frac{|V|^{1 / 2}}{v(., \sqrt{s})^{\frac{1}{r_{2}}}}\right\|_{r_{2}} \frac{d s}{\sqrt{s}}<\infty .
$$

If $N \leq 2$, then the operators $(-\Delta)^{1 / 2} A^{-1 / 2}$ and $|V|^{1 / 2} A^{-1 / 2}$ are bounded on $L^{p}$ for all $p \in(1, r)$, where $r=\inf \left(r_{1}, r_{2}\right)$.

If $N>2$, then $(-\Delta)^{1 / 2} A^{-1 / 2}$ and $|V|^{1 / 2} A^{-1 / 2}$ are bounded on $L^{p}$ for all $p \in\left(p_{0}^{\prime}, \frac{p_{0} r}{p_{0}+r}\right)$.

In particular, if the Riesz transform $\nabla(-\Delta)^{-1 / 2}$ is bounded on $L^{p}$ for all $p \in(2, \delta)$, then $\nabla A^{-1 / 2}$ is bounded on $L^{p}$ for all $p \in\left(p_{0}^{\prime}, \inf \left(\frac{p_{0} r}{p_{0}+r}, \delta\right)\right)$.

Proof: The case $p \in\left(p_{0}^{\prime}, 2\right]$ was already studied in Theorem 3.6, so we consider only $p>2$. We prove that $I-(-\Delta)^{1 / 2} A^{-1 / 2}$ is bounded on $L^{p}$ for all $p$ as in the theorem.

Let $A_{0}:=-\Delta$ and write

$$
\begin{aligned}
I-A_{0}^{\frac{1}{2}} A^{-\frac{1}{2}} & =A_{0}^{\frac{1}{2}}\left(A_{0}^{-\frac{1}{2}}-A^{-\frac{1}{2}}\right) \\
& =C \int_{0}^{\infty} A_{0}^{\frac{1}{2}}\left(I+t A_{0}\right)^{-1} t^{-\frac{1}{2}} d t-C \int_{0}^{\infty} A_{0}^{\frac{1}{2}}(I+t A)^{-1} t^{-\frac{1}{2}} d t \\
& =C \int_{0}^{\infty} A_{0}^{\frac{1}{2}}\left(\left(I+t A_{0}\right)^{-1}-(I+t A)^{-1}\right) t^{-1 / 2} d t
\end{aligned}
$$

Take $\Lambda_{0}$ and $\Lambda$ two operators such that

$$
\mathfrak{a}_{0}(u, v):=<\Lambda_{0} u, v>\text { and } \mathfrak{a}(u, v):=<\Lambda u, v>
$$

where $\langle$,$\rangle is the pairing between D\left(\mathfrak{a}_{0}\right)$ and its dual (or $D(\mathfrak{a})$ and its dual). Note that $D\left(\Lambda_{0}\right):=D\left(\mathfrak{a}_{0}\right)=W^{1,2}$ and $D(\Lambda):=D(\mathfrak{a})=\{u \in$ $\left.W^{1,2}, \int V^{+}|u|^{2}<\infty\right\}$. Here $\mathfrak{a}_{0}$ and $\mathfrak{a}$ are the sesquilinear forms associated with $A_{0}:=-\Delta$ and $A$, respectively. The operator $\Lambda_{0}$ restricted to $D\left(A_{0}\right)$ is $A_{0}$, and $\Lambda$ restricted to $D(A)$ is $A$. Also the restriction of $\left(I+t \Lambda_{0}\right)^{-1}$ to $L^{2}$ coincides with $\left(I+t A_{0}\right)^{-1}$, and the restriction of $(I+t \Lambda)^{-1}$ to $L^{2}$ coincides 
with $(I+t A)^{-1}$. See, e.g., [25] Chapter I.

Set $G(t)$ the following difference:

$$
\begin{aligned}
G(t) & :=A_{0}^{1 / 2}\left(\left(I+t \Lambda_{0}\right)^{-1}-(I+t \Lambda)^{-1}\right) \\
& =t A_{0}^{1 / 2}\left(I+t \Lambda_{0}\right)^{-1}\left(\Lambda-\Lambda_{0}\right)(I+t \Lambda)^{-1} \\
& =t A_{0}^{1 / 2}\left(I+t \Lambda_{0}\right)^{-1} V(I+t \Lambda)^{-1} .
\end{aligned}
$$

Since $D(\Lambda) \subseteq D\left(\Lambda_{0}\right), G(t)$ is well defined. For $f \in L^{2} \cap L^{p}$ we write

$$
G(t) f=t A_{0}^{1 / 2}\left(I+t A_{0}\right)^{-1 / 2}\left(I+t A_{0}\right)^{-1 / 2}|V|^{1 / 2} \operatorname{sign} V .|V|^{1 / 2}(I+t A)^{-1} f .
$$

We estimate the norms of each of the following operators: $A_{0}^{\frac{1}{2}}\left(I+t A_{0}\right)^{-\frac{1}{2}}$, $\left(I+t A_{0}\right)^{-\frac{1}{2}}|V|^{\frac{1}{2}}$, and $|V|^{\frac{1}{2}}(I+t A)^{-1}$.

We begin with the first one. Since $A_{0}$ has a Gaussian upper bound (assumption (3)) we know that $A_{0}$ has a holomorphic functional calculus on $L^{p}$ for all $p \in(1, \infty)$ (see for example [16]). Therefore we have for all $t>0$ and all $p \in(1, \infty)$

$$
\left\|A_{0}^{1 / 2}\left(I+t A_{0}\right)^{-1 / 2}\right\|_{p-p} \leq C t^{-1 / 2} .
$$

In order to estimate the norm of the second operator, $\left(I+t A_{0}\right)^{-1 / 2}|V|^{1 / 2}$, we argue by duality. We write

$$
\left\||V|^{\frac{1}{2}}\left(I+t A_{0}\right)^{-\frac{1}{2}}\right\|_{q-q} \leq\left\||V|^{1 / 2} A_{0}^{-1 / 2}\right\|_{q-q}\left\|A_{0}^{1 / 2}\left(I+t A_{0}\right)^{-1 / 2}\right\|_{q-q}
$$

By Hölder's inequality, we have

$$
\begin{aligned}
\left\||V|^{\frac{1}{2}} A_{0}^{-\frac{1}{2}}\right\|_{q-q} & \leq C \int_{0}^{\infty}\left\|\left.V\right|^{1 / 2} e^{-s A_{0}}\right\|_{q-q} \frac{d s}{\sqrt{s}} \\
& \leq C \int_{0}^{1}\left\|\frac{|V|^{1 / 2}}{v(., \sqrt{s})^{\gamma_{q, q_{1}}}}\right\|_{r_{1}}\left\|v(., \sqrt{s})^{\gamma_{q, q_{1}}} e^{-s A_{0}}\right\|_{q-q_{1}} \frac{d s}{\sqrt{s}} \\
& +C \int_{1}^{\infty}\left\|\frac{|V|^{1 / 2}}{v(., \sqrt{s})^{\gamma_{q, q_{2}}}}\right\|_{r_{2}}\left\|v(., \sqrt{s})^{\gamma_{q, q_{2}}} e^{-s A_{0}}\right\|_{q-q_{2}} \frac{d s}{\sqrt{s}}
\end{aligned}
$$

where $q_{1}$ and $q_{2}$ are chosen such that $\frac{1}{q}-\frac{1}{q_{1}}=\frac{1}{r_{1}}$ and $\frac{1}{q}-\frac{1}{q_{2}}=\frac{1}{r_{2}}$.

Since we assume (3), we have by Proposition 2.9

$$
\left\|v(., \sqrt{s})^{\gamma_{p, q}} e^{-s A_{0}}\right\|_{p-q} \leq C
$$

for $1 \leq p \leq q \leq \infty$. Therefore,

$$
\left\||V|^{\frac{1}{2}} A_{0}^{-\frac{1}{2}}\right\|_{q-q} \leq C \int_{0}^{1}\left\|\frac{|V|^{1 / 2}}{v(., \sqrt{s})^{\frac{1}{r_{1}}}}\right\|_{r_{1}} \frac{d s}{\sqrt{s}}+C \int_{1}^{\infty}\left\|\frac{|V|^{1 / 2}}{v(., \sqrt{s})^{\frac{1}{r_{2}}}}\right\|_{r_{2}} \frac{d s}{\sqrt{s}} .
$$


Using the hypothesis on $V$ we deduce the boundedness of $|V|^{1 / 2} A_{0}^{-1 / 2}$ on $L^{q}$ for all $q \in(1, r)$.

Hence $t^{1 / 2}|V|^{1 / 2}\left(I+t A_{0}\right)^{-1 / 2}$ is uniformly bounded on $L^{q}$ for all $q \in(1, r)$, and by duality

$$
\left\|\left(I+t A_{0}\right)^{-1 / 2}|V|^{1 / 2}\right\|_{p-p} \leq C t^{-1 / 2}
$$

for all $p \in\left(r^{\prime}, \infty\right)$. Thus, using (50) and (52) in (49) we obtain for all $p \in\left(r^{\prime}, \infty\right)$

$$
\|G(t)\|_{p-p} \leq C|||V|^{1 / 2}(I+t A)^{-1} \|_{p-p} .
$$

By the Laplace transform,

$$
\begin{aligned}
\left\||V|^{1 / 2}(I+t A)^{-1}\right\|_{p-p} & =\left\|\int_{0}^{\infty}|V|^{1 / 2} e^{-s} e^{-s t A} d s\right\|_{p-p} \\
& \leq \int_{0}^{\infty} e^{-u / t}|||V|^{1 / 2} e^{-u A} \|_{p-p} \frac{d u}{t}
\end{aligned}
$$

and using (48) we obtain

$$
\begin{aligned}
\left\|I-A_{0}^{1 / 2} A^{-\frac{1}{2}}\right\|_{p-p} & \leq C \int_{0}^{\infty} \int_{0}^{\infty} e^{-u / t}|||V|^{1 / 2} e^{-u A} \|_{p-p} \frac{d u}{t} \frac{d t}{\sqrt{t}} \\
& =C \int_{0}^{\infty}\left|\left\|\left.V\right|^{1 / 2} e^{-u A}\right\|_{p-p} \int_{0}^{\infty} e^{-u / t} t^{-3 / 2} d t d u\right.
\end{aligned}
$$

Since $\int_{0}^{\infty} e^{-u / t} t^{-3 / 2} d t=C u^{-1 / 2}$ we have

$$
\begin{aligned}
\left\|I-A_{0}^{\frac{1}{2}} A^{-\frac{1}{2}}\right\|_{p-p} & \leq C \int_{0}^{\infty}\left\|\left.V\right|^{1 / 2} e^{-u A}\right\|_{p-p} \frac{d u}{\sqrt{u}} \\
& \leq C \int_{0}^{1}\left\|\frac{|V|^{1 / 2}}{v(., \sqrt{u})^{\frac{1}{r_{1}}}}\right\|_{r_{1}}\left\|v(., \sqrt{u})^{\gamma_{p, q_{1}}} e^{-u A}\right\|_{p-m_{1}} \frac{d u}{\sqrt{u}} \\
& +C \int_{1}^{\infty}\left\|\frac{|V|^{1 / 2}}{v(., \sqrt{u})^{\frac{1}{r_{2}}}}\right\|_{r_{2}}\left\|v(., \sqrt{u})^{\gamma_{p, q_{1}}} e^{-u A}\right\|_{p-m_{2}} \frac{d u}{\sqrt{u}} .
\end{aligned}
$$

Here we choose $m_{1}$ and $m_{2}$ such that $\frac{1}{p}-\frac{1}{m_{1}}=\frac{1}{r_{1}}$ and $\frac{1}{p}-\frac{1}{m_{2}}=\frac{1}{r_{2}}$.

Using estimate (46) and the hypothesis on $V$, we have for all $p \in\left(2, \frac{p_{0} r}{r+p_{0}}\right)$

$$
\begin{aligned}
\left\|I-A_{0}^{\frac{1}{2}} A^{-\frac{1}{2}}\right\|_{p-p} & \leq C\left[\int_{0}^{1}\left\|\frac{|V|^{1 / 2}}{v(., \sqrt{u})^{\frac{1}{r_{1}}}}\right\|_{r_{1}} \frac{d u}{\sqrt{u}}+\int_{1}^{\infty}\left\|\frac{|V|^{1 / 2}}{v(., \sqrt{u})^{\frac{1}{r_{2}}}}\right\|_{r_{2}} \frac{d u}{\sqrt{u}}\right] \\
& \leq C .
\end{aligned}
$$


Therefore,

$$
\left\|A_{0}^{\frac{1}{2}} A^{-1 / 2}\right\|_{p-p} \leq C
$$

for all $p \in\left(2, \frac{p_{0} r}{r+p_{0}}\right)$ if $N>2$ and $p \in(2, r)$ if $N \leq 2$.

To obtain boundedness of $|V|^{1 / 2} A^{-1 / 2}$ on $L^{p}$ we write

$$
|V|^{1 / 2} A^{-1 / 2}=|V|^{1 / 2} A_{0}^{-1 / 2} A_{0}^{1 / 2} A^{-1 / 2},
$$

and use the boundedness of $|V|^{1 / 2} A_{0}^{-1 / 2}$ on $L^{p}$ for $p \in(1, r)$ which follows from (51).

As in a previous remark, if $L^{2}$-Poincaré inequality holds and $V^{-}$is such that the heat kernel of $A$ has a Gaussian upper bound like (3), then we obtain in the previous theorem that $(-\Delta)^{1 / 2} A^{-1 / 2}$ and $|V|^{1 / 2} A^{-1 / 2}$ are bounded on $L^{p}$ for all $p \in(1, r)$.

If $v(x, t) \approx t^{N}$ for some $N>2$ and all $t>0$, then the condition on $V$ reads as $V \in L^{N / 2-\varepsilon} \cap L^{N / 2+\varepsilon}$ for some $\varepsilon>0$. Thus if $L^{2}$-Poincaré inequality holds then the conclusion is that $(-\Delta)^{1 / 2} A^{-1 / 2}$ and $|V|^{1 / 2} A^{-1 / 2}$ are bounded on $L^{p}$ for $p \in\left(p_{0}^{\prime}, \frac{p_{0} N}{p_{0}+N}\right)$. Again, in this case $p_{0}=\infty$, then the latter interval is $(1, N)$. For non-negative potentials $V$ that are in a reverse Hölder class, related results are proved in [28], [3] and [6].

\section{References}

[1] Assaad J., Riesz transforms associated to Schrödinger operators with negative potentials, to appear in Publicacions Matemàtiques.

[2] Auscher P., On necessary and sufficient conditions for $L^{p}$-estimates of Riesz transforms associated to elliptic operators on $\mathbb{R}^{N}$ and related estimates, Mem. Amer. Math. Soc.186 no 871, (2007).

[3] Auscher P., Ben Ali B., Maximal inequalities and Riesz transform estimates on $L^{p}$ spaces for Schrödinger operators with nonnegative potentials, Ann. Inst. Fourier, Grenoble 57 no 6, 1975-2013, (2007).

[4] Auscher P., Coulhon T., Riesz transforms on manifolds and Poincaré inequalities, Ann. Sc. Norm. Super. Pisa Cl. Sci. 5 no 4, 1-25, (2005).

[5] Auscher P., Coulhon Th., Duong, X.T., Hofmann S., Riesz transform on manifolds and heat kernel regularity, Ann. Sci. Ecole Norm. Sup. (4) 37, no. 6, 911-957 (2004). 
[6] Badr N., Ben Ali B., $L^{p}$-boundedness of Riesz transform related to Schrödinger operators on a manifold, To appear in Scuola Norm. Sup. di Pisa.

[7] Blunck S., Kunstmann P.C., Weighted norm estimates and maximal regularity, Adv. Diff. Equ. 7 no 12, 1513-1532, (2002).

[8] Blunck S., Kunstmann P.C., Calderòn-Zygmund theory for non-integral operators and the $H^{\infty}$ functional calculus, Rev. Mat. Iberoamericana 19, 919-942, (2003).

[9] Blunck S., Kunstmann P.C., Weak type $(p, p)$ estimates for Riesz transforms, Math. Z. 247, 137-148, (2004).

[10] Blunck S., Kunstmann P.C., Generalized Gaussian estimates and the Legendre transform, J. Operator Theory 53 no 2, 351-365, (2005).

[11] Boutayeb S., Coulhon Th., Sikora, A., in preparation.

[12] Carron G., Coulhon Th., Hassell A., Riesz transform and $L^{p}$-cohomology for manifolds with Euclidean ends, Duke Math. J. 133, no. 1, 59-93 (2006).

[13] Coulhon Th., Duong X.T., Riesz transforms for $1 \leq p \leq 2$. Trans. Amer. Math. Soc. 351, no. 3, 1151-1169 (1999).

[14] Coulhon Th., Sikora A., Gaussian heat kernel upper bounds via the Phragmen-Lindelöf theorem. Proc. Lond. Math. Soc. (3) 96, no. 2, 507544 (2008).

[15] Davies E.B., Simon B., $L^{p}$ norms of non-critical Schrödinger semigroups, J. Funct. Anal. 102, 95-115, (1991).

[16] Duong X.T., Robinson D.W., Semigroup kernels, Poisson bounds, and holomorphic functional calculus, J. Funct. Anal. 142, 89-128, (1996).

[17] Duong X.T., Ouhabaz E.M., Yan L., Endpoint estimates for Riesz transforms of magnetic Schrödinger operators, Ark. Mat. 44, no. 2, 261-275 (2006).

[18] Grigor'yan A., Gaussian upper bounds for the heat kernel on arbitrary manifolds, J. Diff. Geom. 45, 33-52 (1997).

[19] Guillarmou C., Hassell A., Resolvent at low energy and Riesz transform for Schrödinger operators on asymptotically conic manifolds.I, Math. Ann 341 no 4, 859-896, (2008). 
[20] Guillarmou C., Hassell A., Resolvent at low energy and Riesz transform for Schrödinger operators on asymptotically conic manifolds.II, Ann. Inst. Fourier 59 no 4, 1553-1610, (2009).

[21] Kato T., Perturbation theory for linear operators, Grund. der Math. Wiss. 132 Springer Verlag (1966).

[22] Lieskevich V., Semenov, Yu. A., Some problems on Markov semigroups. Schrödinger operators, Markov semigroups, wavelet analysis, operator algebras, 163-217, Math. Top., 11, Akademie Verlag, Berlin, 1996.

[23] Li P., Yau S.T., On the parabolic kernel of the Schrödinger operator, Acta Math. 156, 153-201, (1986).

[24] Liskevich V., Sobol Z., Vogt H., On the $L^{p}$ theory of $C^{0}$-semigroups associated with second-order elliptic operators II, J. Funct. Anal. 193, 55-76, (2002).

[25] Ouhabaz E.M., Analysis of heat equations on domains, London Math. Soc. Monographs 31, Princ. Univ. Press. (2004).

[26] Saloff-Coste L., A note on Poincaré, Sobolev, and Harnack inequalities, Duke J. Math. 65, 27-38,I.R.M.N. (1992).

[27] Sikora A., Riesz transform, Gaussian bounds and the method of wave equation, Math. Z. 247, 643-662, (2004).

[28] Shen Z., $L^{p}$ estimates for Schrödinger operators with certain potentials, Ann. Inst. Fourier (Grenoble) 45, no. 2, 513-546, (1995).

[29] Takeda M., Gaussian bounds of heat kernels for Schrödinger operators on Riemannian manifolds, Bull. London Math. Soc. 39, 85-94, (2007). 